\title{
Mitteilungen aus dem Pharmazeutisch-chemischen Institut der Universität Marburg.
}

\section{Ueber die Synthese des inalktiven Ephedrins bez. Pseudoephedrins.}

\author{
Von Dr. August Eber hard.
}

Unter den wenigen in der Natur vorkommenden Alkaloiden mit sekandärem Amincharakier stehen Ephedirin und Pscudoephedrin, denen beiden die Formel $\mathrm{C}_{10} \mathrm{H}_{15} \mathrm{NO}$ zukommt, an erster Stelle. Mit ihrer Lrforschung hat sich neben $\left.\mathrm{Nag} \mathrm{a} \mathrm{i}{ }^{1}\right), \mathrm{M} \mathrm{er} \mathrm{rk}^{2}$ ), Ladenburg und Oolsehlägel ${ }^{3}$, Fournea a und R a b e ${ }^{5}$ ), hauptsä.hlich F. Sehmidt.6) mit seinen Schülern, za denen auch ich mich zählen darf, seit etwa zwei Dezennien beschäftigt. Beide Alkaloide besitzen eine Phenyl-, eine nicht endständige Methylimidgruppe sowie eine Alkoholaruppe, welche durch den Verlauf der H of ma nn'schen Spaltung als sekundäre Alkoholgruppe gekennzeichnet ist. Weiter läßt das qualitativ und quantitativ versehiedene Drehungsvermögen sowie die reversihle Umlagerungsfähigkeit der Basen auf das Vorhandensein zweier asymmetrischer Kohlenstoffatome schließen, so daß ein Fall der Diasiereomerie vorliegt. Für die Konstitution der Alkaloide kommen demnach vier optisch aktive Formeln in Frage; neben diesen ist die Bildung zweier racemischer Verbindungen möglieth. Anf Grund seiner Betrachtungen über die Haftfestugkeit der C-N-Bindung sowie theoretischer Ueberlegungen orteilt E m d e ?) beiden Basen dio Formel $\mathrm{C}_{6} \mathrm{H}_{5} \mathrm{CH}\left(\mathrm{NHCH}_{3}\right) \mathrm{CHOHCH}_{3}$ und vermutet als Grund dor reversiblen Umlagerang des Ephedrins in das Pseudoephedrin ein "Umklappen" der Methylimidgruppe. $\mathrm{Zu}$ derselben Formel gelangt $\mathrm{G}$ a d a $\mathrm{m}$ e ${ }^{8}$ ) auf rein theoretischem
1) Chem.-Ztg. 1890, I.. 441 .
2) Merck's Berichte 1893, 13; 1894, 177 .
s) Berichte 22 (1889), 1823 .
1) Tourn. de Pharm. et de Chim. XX (1904), 481 ; XXV (1907), 氵993.
-) Berichto 44 (1911), 824.
-) E. R. M i l 1 o r, Areh. d. Pharm, 240 (1902), 481. F l ä e h e r, ibid. 242 (1904), 382. F, S c h m i d t, ibid. 243 (1905), 73; 944 (1906). $240 ; 246$ (1908), 210; 247 (1909), 141; 249 (1911), 221, 305; 251 (1913), 320 ; 252 (1914), 89 ; 253 (1915), 52. H. E m d e ibid. 244 (1906), 241, $269 ; 245$ (1907), 662; 247 (1909), 55, 137; 249 (1911), 354; Ber. 43 (1910), 1727. Ga d a $\mathrm{m}$, Arch. d. Pharm. 246 (1908), 560. $\mathrm{B}$ ï m m in g, Inaug.-Dissert. Marburg 1909. G o $\Theta \mathrm{h} \mathbf{r}$ ing, Arch. d. Pharm. 247 (1909), 145. C a l l i o B, Inaug.-Dissert. Marburg 1912; Arch. d. Pharm. \$气0 (1912). 164; Apoth.-Ztg. 1910. No. 72. H b e r h a r d, Inaug.-Dissert. Marburg 1914; Arch. d. Pharm, 253 (1915), 62.

7) Arch. d. Pharm. 245 (1907), 662.

-) Arch. d. Pharm. $\mathbf{9 4 6}$ (1908), 566. 
Wege, weist aber auf die größere Wahrscheinlichkeit der Alkoholinversion hin bei der Umlagerung Ephedrin $\rightleftarrows$ Pseadoephedrin.

Im Gegensatz zu dieser Annahme schreibt E. Sch midt (1. c.) den Alkaloiden die Formel: $\mathrm{C}_{6} \mathrm{H}_{5}\left(\mathrm{H}(\mathrm{OH}) \mathrm{CH}\left(\mathrm{NHCH}_{3}\right) \mathrm{CH}_{3}\right.$, zu. Hierzu veranlaßt ihn einmal die glatte Bildang großer Mengen von Benzaldehyd bei der Oxydation der Alkaloide mit Ferricyankalium, Permanganat and den Halogenen, die darauf hinweist, daß die $\mathrm{OH}$-Gruppe an einem dem Benzolring benachbarten Kohlenst offatom steht, andererseits die (vermutlich sekundäre) Bildung von Aethylphenylketon neben Phenylpropylenoxyd bei der Spaltung der salzæauren Salze im Kohlensüurestrom. Durch die gleich starke optische Aktivität (Rechtsdrehung) des aus beiden natürlichen Basen durch Einwirkung von Phosphorpentabromid und darauffolgende Reduktion erhaltenen Phenylmethylaminopropans, die darch Behandeln mit Salzsïure keine Veränderung erfïhrt, beweist er, daß die Methylimidgruppe bei der Umlagerung der Alkaloide umbetriligt ist, vielmehr die OH-Gruppe allein an der Inversion beteiligt ist.

Daraus folgt mit großer Wahrscheinliehkeit, daß im Ephedrin je ein rechts- und ein linksdrehendes asymmetrisches Kohlenst offatom, im Pseudoephedrin dagegen zwei rechtsdrehende Gruppen vorhanden sind. In der Annahme, daß den natürlichen Alkaloiden die Formelbilder I und II zukommen, müssen die Formeln IIJ und IV den bisher unbekannten Spiegelbildisomer en entsprechen, während die Vereinigung von I und III sowie II und IV die ebenfalls unbekannten Racemformen zum Ausdruck bringen, wie sie analog von $\mathrm{E} \mathrm{m} \mathrm{de}$ (l. c.) angegehen sind.

I.

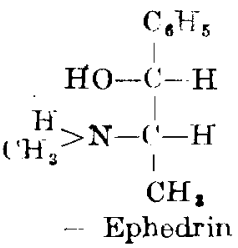

III.

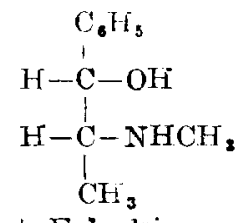

II.

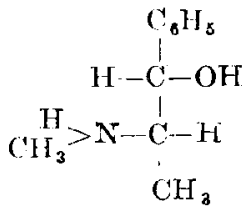

+ Pseudoephedrin

IV.<smiles>CCCCCCC(C)NCC</smiles>

- Pseudoephedrin

unbekannt.

Obwohl ein experimenteller Beweis für die Richtigkeit der anfgestellten Formeln noch heute aussteht - die dahingehenden Versuche, einmal die naturellen, optisch aktiven Basen zu racemisieren. und zum anderen eine Spaltung der synthetischen Verbindungen 
obiger Konsíitution durchzuführen, sind bisher erfolglos geblieben -, setzen schon frühzeitig Versuche zur Synthese dieser Verbindıng ein, und zwar lassen sich diese Versuche in zwei Gruppen scheiden.

Die Mehrzahl der Antoren strebt die Synthese einer ephedrinartigen Verbindung durch Einführung der Methylamjdogruppe in einen entsprechenden Komplex an. Schon 1904 hat Fourne a u (I. c.) neben anderen Is meren, die dem Ephedrin bzw. dem Pseudoephedrin absolut unähnlich waren, eine Verbindung obiger $7 / u^{-}$ sammensetzung durch Einwirkung von Methylamin auf das Jodhydrin des Phenylpropylens gewonnen, die den Gervch des Pseuloephedrins und einige ihm nahestehenden Eigenschaften aufwies. Denselben Weg schlug $\mathrm{B}$ ü $\mathrm{m} \mathrm{m}$ i $\mathrm{n} g 1909$ (s. o.) ein und vermutete in der erhaltenen Verbindung die inaktive Form der naturellen Base. Weiter haben damn $\mathrm{R} a \mathrm{~b}$ e und $\mathrm{H}$ a 11 e $\mathrm{n} \mathrm{s}$ le be $\mathrm{n}^{1}$ ) dureh Einwirkung von Methylamin auf Propylenoxyd die gleiche Verbindung dargestellt. S.hließlich habe ich ${ }^{2}$ ) auf Veranlassung von Herrn Geh. Reg.-Rat E. S c h mid t den von Ca lli e $B^{3}$ ) erfolslos begonnenen Versuch, durch Einwirkung von Methylamin auf $\alpha$-Brompropiophenon und nachträgliche Reduktion zu einem synthetischen Ephedrin-Isomeren zu gelangen, aufgenummen. Durch Analyse des salzsauren Salzes sowie der Platin-Doppelverbindung konnte das Reaktionsprodukt als Verbindung der Zusammensotzung ${ }_{10} \mathrm{H}_{15} \mathrm{NO}$ gekennzeichnet werden. Auch wurde die freie liase isoliert und ihr Schmelzpunkt $114^{\circ}$ bestimmt. Eine eillsrehendere Charakterisierung des Reaktionsproduktes int mir indessen infolge der geringen Ausbeute ebensowenig möglich gewesen wie seineszeit $\mathrm{B} \ddot{\mathrm{u}} \mathrm{m} \mathrm{m}$ ing (l. c.).

Der zweite, nicht wesentlich verschiedene Weg führt theoretisch leicht über das $\alpha$-Amidoäthylphenylketon, einmal durch Einführung einer Methylgruppe und nachträgliche Reduktion zum Methylamidoäthylphenylcarbinol, zum anderen in umgekehrter Reihenfolge - zunächst Reduktion des Amidopropiophenons z!l dem beständigen Phenylamidopropanol und darauffolgende Methylierung - zu dem gesuchten Produkt. Auf diesem Weg hat sich rinerst Ca l I i e B (a. a. O.) versucht, und zwar in beiden Richtungen. Indessen ist es ihm nicht gelungen, ein Monomethylierungsprodukt durch Einwirkung von Dimethylsulfat auf $\alpha$-Amidopropiophenon oder von Jodmethyl auf das durch Reduktion aus diesem Amirioketon erhaltene Amidocarbinol zu i:olieren. In beiden Fällen resultierten stets nur tertiäre und quartäre Verbindungen, die eigentümlicherweise mit einer aus Trimethylamin und Brompropiophenon dargestellten Verbindung nicht übereinstimmten. Auch in dieser Richtung bin ich in meiner Inaugural-Dissertation den Fußtapfen Ca llie 3 ' gefolgt, und zwar mit demselben negativen Erfolg. Weder Diazomethan noch Dimethylsulfat noch Jodmethyl wirkten in dem gewïnschten Sinne auf das Amidoketon ein. Ebenso waren durch Einwirkung von Jodmethyl auf das Amidoäthylphenyl-

1) Berichte 44 (1911), 824.

2) Inaug.-Dissert. Marburg 1914.

3) Apoth.-Ztg. 1911, No. 37 . 
carbinol wohl die tertiäre und die quaternäre Verbindung gewonnen worden, dagegen war es auch mir nicht gelungen, das gesuchte Monomethylamidoä hylphenylcarbinol auf diesem Wege darzustellen.

Seiłdem habe ich mich mit weiteren Versuchen zur Synthese des Ephedrins beschäfigt und zwar habe jch — mit Rücksicht auf die geringe Ausbeute an dem bisher durch Einwirkung von Methylamin auf $\alpha$-Brompropiophenon gewonnenen Reaktionsprodukt -zunächst versucht, auf dem theoretisch am einfachsten und aussichtsreichsten erscheinenden Weg über das Amidoäthylphenylketon bzw. das entsprechende Amidocarbinol zu einem synthetischen Ephedrin zu gelangen. Dieser Weg mußte um so gangbarer erscheinen, als es mir inzwischen ${ }^{1}$ ) gelungen war, das Amidopropiophenon auf katalyischem Weg mit Hilfe von Palladiumkohle glatt in das Amidoäthylphenylcarbinol überzuführen und so letztere Verbindung in gui ser Ausbeute und größercr Reinheit darzustellen als dies seinerzeit durch Finwirkung von Natriumamalgam in saurer Lösung möglich war. Als Methylicrungsmittel wurden Dimethylsulfat, Methylalkohol, Jodmethyl und Formaldehyd unter verschiedenen Bedingungen angewandt. Diese Versuche haben das gesuchte Reaktionsprodukt bis auf einen cinzigen Fall nicht geliefert. Dagegen resultiente bei der Einwirkung von Dimethylsulfat auf das salzsauere Carbinol in der Wärme ebenso wie auf die freie Amidobase in der Kälue eine Verbindung von betainartigem Charakter, die mit der von E. S c h m i d t ${ }^{2}$ ) durch Erwärmen von Schwefelsäure und Ephedrin bzw. durch Zusammenbringen von Pseudoephedrin und Silborsulfat erhaltenen Verbindung der $\mathrm{Zu}$ sammensctzung $\mathrm{C}_{10} \mathrm{H}_{10} \mathrm{NO} . \mathrm{SO}_{3}$ große Aehnlichkeis im Aeußeren, ihrem Schmelzpunkt und ihrem sonstigen Verhalien besitzt, deren Spaltungsprodakt aber auf $\mathrm{n}$ ich $\mathrm{t}$ mothyliertes Amidocarbinol hindeutet. Andererseits entstand bei Methylierungsversuchen des Amidoketons das Dimethyldiphenylpyrazin, $\mathrm{C}_{18} \mathrm{H}_{16} \mathrm{~N}_{. .}$, das durch seine Silber-, Gold- und Platindoppelsalze sowie durch sein Hydrochlorid charakterisiert wurde.

Eine Erklïrung finden diese, auch von anderer Seite ${ }^{3}$ ) gemachten negativen Erfahrungen in der von $W$ en $z$ el aufgestellten Theorie des zweikernigen Stickstoffatoms. Nach $W$ e n z e l tritt bei der Bildung von Monalkylbasen das Radikal an den $\mathbf{N}-\alpha$-Kern, das Halogen an den $\mathbf{N}-\beta-K e r n$, und so bildet sich ein stabiles Salz, das bei Ausschluß hydrolytischer Spaltung nicht weiter verändert wird. Bei enneuter Einwirkung von Halogenalkyl auf ein freies Monalkylamin tritt jetzt das Radikal in $\beta$ Siellung, das Halogen an den weniger basischen $N$ - $\alpha$-Kern. Aus dem so entstandenen Salz der schwachen Dialkylamin- $\alpha$-Base sełzen die noch unveränderten sïärkeren Monalkylbasen erstere in Freiheit, so daß von neuem Halogenalkyl einwirken kann, bis

1) Arch. d. Pharm. 255 (1917), 142.

2) ibid. 252 (1914) 124.

3) Em d e, Arch, d. Pharm. 247 (1909), 354.

4) Monatshet te d. Chem. XXXVIII (1917), 267. 
schließlich nach nochmaliger Umsetzung mit dem Alkylierungsmittel quaternäre Verbindungen resultieren.

Auch die Versuche, durch Absättigung eines Wasserstoffatoms der Amidogruppe das restierende Wasserstoffatom für die Substitution durch Methyl geeigneter zu machen, haben sich auf dem Weg über die Acetyl- und die Benzoylamidoverbindungen als erfolglos erwiesen, da durch den Eintritt der Süuregruppe der basische Charakter der Verbindung derartig geschwächt wurde, daB Jodmethyl selbst unter Druck nicht mehr einzuwirken vermochte. Schließlich hat sich auch die Anwendung von Benzaldehyd in Kombination mit nachfolgender Methylierung in der Mehrzahl der Fälle als ungangbar erwiesen. Nur in einem einzigen Fall resultierten geringe Mengen eines Produktes, das als ephedrinartige Verbindung gekennzeichnet werden konnte.

In Anbetracht der Ungangbarkeit dieses Weges blieb nur die Möglichkeit, auf die bereits früher (l. e.) angegebene Weise durch Einwirkung von Methylamin auf $\alpha$-Brompropiophenon und nachfolgende Reduktion zu der gesuchten ephedrinähnlichen Verbindung zu gelangen and so genügend Material zu eingehenden Untersuchungen djeser synthetischen Base zu gewinnen. Mit dieser Aufgabe, der Identifizierung des Reaktionsproduktes sowie mit Umlagerungsversuchen befaßt sich der zweite Teil meiner Arbeit. Im Gegensatz zu der früher stets schlechten Ausbeute bei der Reduktion des Methylamidopropiophenons mit Natriumamalgam in saurer Lösung wurde jetzt durch Einführung der erwähnten katalytischen Hydrierung das Methylamidoäthylphenylcarbinol in befriedigender Ausbeute in reinem Zustand gewonnen. Hierbei resultierte in der Hauptsache eine durch gute Krystallisierbarkeit ihres Hydrochlorides sowie durch zahlreiche andere Eigenschaften von der früher schon isolierten Base abweichende Verbindung, die durch Einwirkung von Salzsäure und durch Acetylierung eine Umlagerung in die früher erhaltene Form erfährt, wie sie ähnlich E. Sch m i d mit den naturellen Basen durchgeführt hat.

Die Durchführung der in folgenden experimentellen Teil beschriebenen Versuche, die bis auf wenige A,brundungen im Pliarmazeutisch-chemischen. Institut der Universität Marburg ausgeführt sind, wurde durch die zeitweilige Gassperre wesentlich ersithwert. So war ich häufig genötigt, Fragen, die durch Ausführung von Elementaranalysen leicht häiten geklärt werden können, auf anderem Wege zu entscheiden.

Die Analysen der Gold- und Platindompelsalze wurden stets mit Hilfe von Schwefelwasserstoff ausgefühtt, um eine Rückgewinnung des Materials zu ermöglichen. 


\section{Versuche zur Synthese ephedrinartiger Verbindungen durch Methylierung des Amidopropiophenons bzw. des Amidoäthylphenylcarbinols.}

\section{Methylierungsversuche mit Dimethylsulfat.}

Wie eingangs erwähnt, ist bisher eine Methylierung des a-Amidoäthylphenylcarbinols nur mit Hilfe von Jodmethyl durchgefïhrt worden, ohne daß es jedoch dabei gelungen wäre, eine Monomethylverbindung zu isolieren. Es wurde daher zunächst versucht, unter Alwendung ron Dimethylsulfat als Methylierungsmittel $z u$ der gewünschten ephedrinähnlichen Verbindung zu gelangen. Da jedoch anch dieses Alkylierungsmittel bei dem I-Phenyl2-amidoprojaanon (1) nur teriüre und quaternäre Verbindungen geliefert hatte, so war es eiforderlich, durch Abänderung der Versuchsbedingungen, eine Beeinflussung des Reaktionsverlaufes in dem gewünschten Sinn zu erzielen.

Zur Erreichungr dieses Zieles wurde zunächst $1 \mathrm{~g}$ salzsaures Amidoäthylphenylcarbinol in feinzerriebenem Zusiand mit $4 \mathrm{~g}$ Dimethylsulfat auf dem Wasserbad am Rückflußkïhler erhitzt. Sobald nach etwa 15 Minuten das Pulver in Tuösung gegangen war, wurde eine Probe der Flüssigkeit entrommen (I) und für sich untersucht. Die Hauptmenge des Reaktionsgemisches wurde dann weiter erhitzt, wodurch es nach Verlauf von weiteren I0 Minuten zu einer schneeweißen Masse (II) erstarrte.

Die dem Realitionsproduki zunächst entnommene Probe (I) wurde durch Einengen mit konzentrierter Salzsïure von überschüssigem Dimethylsulfat befreit und der hierbei verbleibende Rüekstand alsdann mit Golkchloridlösung versetzt. Die hierbei hauptsächlich resultierenden rotgelben Warzen vom F. $165^{\circ}$ erwiesen sich als ein schwach rerunreinigtes Colddoppelsalz des Ausgangsmaterials (F. $172^{\circ}$ ).

$0,2368 \mathrm{~g}$ des im Vakumu getrockneten foldsakes lieferten $0.0962 \mathrm{~g}$ Gold.

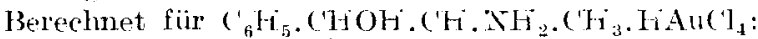
Ali 40,12

Gefunden : $40.70 \%$

Daneben resultierten nur in geringer Ausbente zitronengelbe Nadeln, die unscharf zwischen 115 und $120^{\circ} \mathrm{zu}$ eincr trüben Flüssigkeit schmolzen. Ein aus den Mutterlangen dargestelltes Platinsalz zeigte den F. $180^{\circ}$ unter Aufschäumen; indessen reichte seine Menge nicht zu einer weiteren Charakterisierung aus. Der Schmelzpunkt dieses Gold- und ebenso der des Platinsalzes erimnern an das Verhalten der entsprechenden Doppelverbindungen des Phenylmethylamidopropanols. Eine Entseheidung, ob die gestachte Verbindung vorlag, lieb sich indessen infolge der geringen Ausbente nicht herbeiführen. Jedenfalls hatte aber die Untersuchung ergeben. daß die Reaktion zur Zeit der Probeentnahme noch nicht genügend vorgeschritten war.

Um so aussichtsreicher mußte die Zerlegung des durch weiteres Erhitzen gewonnenen festen Reaktionsproduktes (II) erseheinen. Dieses war in Alkohol nicht völlig lüslich. Erst ein Zusatz von Wasser und Salzsïure brachte die letzten Anteile in Iösung, aus 
A. Eberhard: Inaktives Ephedrin bez. Pseudoephedrin. 103

ler sich beim Erkalten farblose, stark glänzende Nadeln und Blättchen ausschieden, deren F. unter Aufschäumen bei $244^{\circ}$ lac̆. Die Krystalle verhielten sich indifferent gegen Lackmus, Silbernitrat und Chlorbaryum. Erst durch Kochen mit konzentrierter Salzsäure wurde der in der Verbindung enthaltene Schwefelsäurerest als solcher ionisiert. Da die Bestandigkeit dieses Reaktionsproduktes selbst beim Erhitzen auf $150^{\circ}$ das Vorliegen eines methylschwefelsauren Salzes unwahrscheinlich machte, schien es sich im eine betainartige Verbindung $z u$ handeln, wie sie von $W$ ill st it ter und $\left.H u g^{3}\right)$ bei Atropin und Scopolamin beobachtet wurde, und wje sie ähnlich $\mathrm{E}$. Se h $\mathrm{mid} \mathrm{t}{ }^{2}$ ) aus dem Ephedrin durch Einwirkung von konzentrierter Schwefelsäure und aus dem Psendoephedrin durch Umsetzung mit Silbersulfat erhalten hat. Da das A,eußere, der Schmelzpunkt und das Verhalten gegen Chlorbaryum mit dem cler Ephedrinabkönmlinge übereinstimmt, anderersei:s aber die Analysonwerte ergeben, daß die erwartete Methylierung nicht eingetreten war, so dürfte der Verbindung die Formel

zukommen.

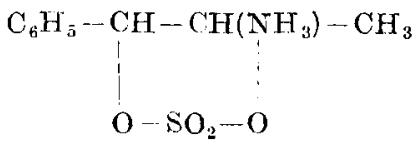

1. $0,1837 \mathrm{~g}$ ergaben $0.3138 \mathrm{~g} \mathrm{CO} \mathrm{CO}_{2}$ und $0.0900 \mathrm{~g} \mathrm{H}_{2} \mathrm{O}$.

2. $0,1867 \mathrm{~g}$ ergaben $0,3211 \mathrm{~g} \quad \mathrm{CO}_{2}$ und $0,0956 \mathrm{~g} \mathrm{H}_{2} \mathrm{O}$.

3. $0,2148 \mathrm{~g}$ ergaber $0.2174 \mathrm{~g} \mathrm{BaSO}_{4} \mid$ Dureh einstündiges Kuchen mit

4. $0,6465 \mathrm{~g}$ ergaben $0,6620 \mathrm{~g} \mathrm{BaSO}_{4}$

5. $0,1922 \mathrm{~g}$ ergaben $0.194 \mathrm{~g} \mathrm{BaSO}_{4} \mathrm{~J} \quad \mathrm{HCl}$ gespalten.

6. $0.1960 \mathrm{~g}$ ergaben $0.201+\mathrm{g} \mathrm{BaSO}_{4}$ (wach ( a rius).

Berechnet fïr:

$\begin{array}{cc}\mathrm{C}_{0} \mathrm{H}_{11} \mathrm{NO}_{4} \mathrm{~s} & \mathrm{C}_{9} \mathrm{H}_{13} \mathrm{NO}_{4} \mathrm{~s} \\ 47.14 & 46.73 \\ \mathrm{H} 4.84 & 5.67 \\ \mathrm{H} 13.98 & 13,87\end{array}$

Gefunden:

\section{Das bei clen ischwefelsatrebestim} S Das bei ten schwefelsäurebestimmungen $3-5$ resultierende Spaltungsprodukt konnte durch Ueberführung in das salzsaure Salz (farblose Blättchen vom F. 1919) und in das Goldsalz (rotgelbe Krystalle, F. 1720) sowie durch die Analyse der bei $197^{\circ}$ unter Aufschäumen schmelzenden Platindoppel verbindung als nichtmethyliertes Amidoäthylphenylcarbinol gekennzeirchnet werden.

$0,4100 \mathrm{~g}$ verloren bei $100^{0} 0.0121 \mathrm{~g}$ an Gewicht.

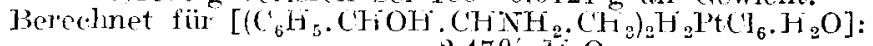

$$
\begin{gathered}
2,47 \% \mathrm{H}_{2} \mathrm{O} \\
\text { Gcfunden : } \\
2,95 \%
\end{gathered}
$$

$0,3979 \mathrm{~g}$ des getrockneten 1'latinsalzes ergaben $0,1092 \mathrm{~g}$ Pt. Bereclint für

$\left[\left(\mathrm{C}_{6} \mathrm{H}_{5} \cdot\left(\mathrm{HOH}\right.\right.\right.$. ( $\mathrm{H}$. $\left.\mathrm{NH}_{2} .\left(\mathrm{H}_{3}\right)_{2} \mathrm{H}_{2} \mathrm{PtCl}_{6}\right]$ :

$$
27,38 \% \text { \% }
$$

Gefunden:

$27.44 \%$

i) Ztsehr'. f. physiolog. (hemie 79,155 .

2) Arch. d. 1'hirm. Et: (1914), 124. 
Dieselbe betainartige Verbindung resultierte neben unverändertem Ausgangsmaterial auch bei dreitägigem Stehen des Reaktionsgemisches bei Zimmertemperatur.

Auch die Versuche, durch Einwirkung $\checkmark$ on Dimethylsulfat auf das freie Amidoäthylphenylcarbinol, unter Beschränkung der Mengen des Methylierungsmittels, das gewünschte Ziel zu erreichen, waren ohne Erfolg.

In Anlehnung an das S e h ot te en - B a u $\mathrm{m}$ a n n'sche Benzoylierungsverfahren wurden zu diesem Zwecke zunächst $1,87 \mathrm{~g}$ des salzsauren Amidocarbinols in $20 \mathrm{~g} 15 \%$ iger Natronlauge fein verteilt und diesem Gemisch dann unter Umschütteln tropfenweise $0,63 \mathrm{~g}$ Dimethylsulfat zugesetzt. Außer einer Gelbfärbung der von dem Dimethylsulfat getroffenen Amidocarbinol-Teilchen war indessen keine Veränderung des Materials feststellbar. Die nach halbstündigem Stehen des Peaktionsgemisches durch Aether bzw. Chloroform isolierte freje Base zeigte den F. $101^{0}$ sowie alle übrigen Eigenschaften der unveränderten freien Amidobase. Auch durch Ueberführen in das bei $189^{\circ}$ schmelzende Hydrochlorid ließ sich dieses Produkt als Ausgangsmaterjal charakterisieren.

Weiter wurde versueht, durch Erwärmen der freien Amidobase mit Dimethylsulfat in einem indifferenten Lösungsmittel zum Ziel zı gelingen. 1,05 g des frisch dargestellten Amidocarbinols wurden zu cliesem Zwer:k in Aether gelöst und diese Lösung dann mit $0,43 \mathrm{~g}$ Dimethylsulfat zwei Stunden lang am Rückflußkühler erhitzt. Hierbei schieden sich an den Wandungen ölige Tröpfchen ab, die nach dem Erkalten weiße Nadelgruppen einschlossen.

Die von diesen Abseheidungen getrennte ätherische Lösung hinterließ beim Verdunsten einen obligen Wandbelug. der - znu Trennung der Bestand teilo mit Goldrhlorid versetzt - in der Hauptsache schwerlösliche, veristelte Nadeln vom F. 128 bis $129^{\circ}$ lieferte. Der Goldgehalt dieser Verbindung stimmt nit dem eines tertiären Methylamindoppelsalzes überein.

$0,1849 \mathrm{~g}$ der exsikkatortrocken $\mathrm{n}$ Suhstanz ergaben $0.0704 \mathrm{~g}$ (hild. Berechnet für $\mathrm{C}_{6} \mathrm{H}_{5} . \mathrm{CHOH} . \mathrm{CH} . \mathrm{Nu}\left(\mathrm{CH}_{3}\right)_{2} . \mathrm{CH}_{3} . \mathrm{HAMCl} \mathrm{CH}_{4}$ : Gefunden:

Aus den Mutterlaugen resultierten zunichst in geringor Menge rotgelbe Warzen und Krrusten vom F. 152-1550 und schlicßlich -hellgelbe Nadeldrusen, die bei $105-115^{\circ}$ unscharf schmolien. Letztere Aussrcheidungen wurden zusammen mit den weiter unten. erwähnten glejchartigen Krystallen verarbeitet.

Auch der tröpfchenartigo Wandbeliag warde in Goldsalze verwandelt. Neben geringen Nengen selıwerer löslicher zitronengelber Lamellen vom F. $173^{\circ}$ lieferte er in der Hauptsache jedoch nur die Doppelverbindung des Ausgangsmaterials (rotgelbe warzenartige Gebilde vom F. 172\%.

$$
\begin{aligned}
& 0,388 \mathrm{~g} \text { de's in Valiuum getrockneten Guldsalzes liefelten } \\
& 0.1566 \mathrm{~g} \text { G } \mathrm{i}: 1 \mathrm{ld} \text {. } \\
& \text { Berechnet für } \mathrm{C}_{9} \mathrm{H}_{13} \mathrm{NOO} . \mathrm{HAuCl}_{4} \text { : } \\
& \text { Au } 40,12 \\
& \text { Gefunden: } \\
& 40,35 \%
\end{aligned}
$$


Das Filtrat dieser Bestimmung diente zur Darstellung eines Platinsalzes. Dasselbe bildete matte, orangefarbene, verfilzte Nadelbüschel, die bei $187-191^{\circ}$ unter Aufschäumen schmolzen.

Die weiter ebenso wie aus der ätherischen Lösung (s. o.) resultierenden hellgelben flachen Nadeln und Blättchen mit unscharfem, niedrigem Schmelzpunkt ließen sich nicht genügend reinigen (Goldgehalt 40,72\%). Ebensowenig gelang es, diesen Teil des Reaktionsproduktes durch Ueberführung in ein Platindoppelsalz als einheitliches Produkt zu isolieren. Wenn auch das Aeußere und der niedrige Schmelzpunkt des letztgenannten Golddoppelsalzes eine gewisse Uebereinstimmung mit dem Chloroaurat des Methylamidocarbinols aufweist, so zeigt doch andererseits die geringe Ausbeute, daß die gewünschte Reaktion unter obigen Bedingungen in der Hauptsache nicht eingetreten ist.

In der Annahme, daß bei diesem Versuch das Lösungsmittel (Ae'her) die Reaktion verzögert habe, wurden nummehr $0,5 \mathrm{~g}$ der freien Amidoverbindung direkit mit überschüssigem Dimethylsulfat versetzt. Unter starker Temperaturerhöhung und lebhaftem Aufschïumen resultierte hierbei eine Flüssigkeit, die beim Erkalten Krystalle abschied. Nach viertägigem Stehen vermochte Aether dem Reaktionsprodukt nur Dimethylsulfat, aber keine freie Base mehr zu entziehen. Bei der Ueberführung des krystallisierten Anteils in ein Goldsal\% wurden zunächst glänzende gelbe Schuppen gewonnen, deren Goldgehalt auf eine tertiäre Verbindung hinwies, die aber im Gegensatz zu dem bei dem vorigen Versuch erhaltenen tertiären Aurat bei $179^{\circ}$ schmolz.

$0,0860 \mathrm{~g}$ ergaben $0,0327 \mathrm{~g}$ Gold.

Berechnet für $\left(\mathrm{C}_{6} \mathrm{H}_{5} . \mathrm{CHOH} \cdot \mathrm{CH} \cdot \mathrm{N}\left(\mathrm{CH}_{3}\right)_{2} \cdot\left(\mathrm{CH}_{3} \cdot \mathrm{HAuCl}_{4}\right)\right.$ : Gefunden: Au 38.00 $38,02 \%$

Die beim Einengen Benzaldehydgeruch aufweisenden Mutterlaugen lieferten, ebenso wie bei dem vorigen Versuch, gelbe Nadeln und Blättchen, die unscharf zwischen $87^{\circ}$ und $100^{\circ}$ schmolzen.

Die Methylierungsversuche mit Dimethylsulfat hatten somit nicht oder doch nur in so ungenügenden Mengen ein Methylierungsprodukt geliefert, daß dessen Identitait mit dem gesuchten Methylamidoäthylphenylearbinol nicht bewiesen werden konnte. Lediglich die nicht methylierte betainartige Verbindung de: Zusammensozung $\mathrm{C}_{9} \mathrm{H}_{13} \mathrm{NO}_{4} \mathrm{~S}$ trat hierbei in größerem Umfang auf.

Da bei den früher ausgeführten Methylierungsversuchen des Amidoketons die Entstehung eines entsprechenden Produktes mit betainartigem Charakter nicht beobachtet wurde, so muß die Bildung diese: Verbindung erst nach der Reduktion der Ketongruppe zur sekundären Alkoholgruppe ermöglicht werden. Zur Kontrolle dieser Annahme wurde daher das Verhalen des Amidoäthylphenylketons gegen Dimethylsulfat unter den gleichen Versuchsbedingungen nachgeprüft.

Mit Rücksicht auf die geringe Beständigkeit des freien Amidopropiophenons wurden $1,85 \mathrm{~g}$ des salzsauren Salzes in $50 \mathrm{ccm}$ Wasser gelöst, die Lösung mit $0,63 \mathrm{~g}$ Dimethylsulfat versetzt und dem Gemisch unter Uimsohwenken tropfenweise $10 \mathrm{ccm}^{1} / 10^{-\mathrm{N}}$.-Kalilauge zugegeben. Die Ï̈sung nahm durch den Langenzusatz eine Gelb- 
färbung an, trübte sich mul wurde schließlich undurchsichtig. Nach halbstündigem Stehen ließcn sich dem Reaktionsgemisch Trübung und Färbung durch Aether entziehen.

Bei der Aufarbeitung dieses ätherischen Aluszuges wurde nur ein bei $95-98^{\circ}$ schmelzendes Quecksilberdoppelsalc isoliert, dessen Metallgehalt auf nonverändertes Ausgangsmaterial hinwies.

$0,4979 \mathrm{~g}$ verloren in Exsilskator $0,0127 \mathrm{~g}$.

$0,4852 \mathrm{~g}$ exsilikatortrokene Substanz licferten $0.1702 \mathrm{~g} \mathrm{HgS.}$

Berechnet für $\left(\mathrm{C}_{2} \mathrm{H}_{11} \mathrm{NO} . \mathrm{H}\left\{\mathrm{Cl}, \mathrm{HgCl}_{2} . \mathrm{H}_{2} \mathrm{O}\right)\right.$ : Gefunden:

$$
\begin{array}{lr}
\mathrm{H}_{2} \mathrm{O}=, 72 & 2,55 \% \\
\mathrm{Hg} 30,95 & 30,23 \%
\end{array}
$$

Die mit Aether ansgoschüttelte wässerige Flüssigkeit wurde nach Zusatz ron Salzsiture zur Trockne rerdampft. Der alkoholische Auszug dieses Trockenr ückstandes lieferte cinen aus seidenglänzenden Nadeln bestehenden, leichtlöslichen Wandbelag, dessen Golddoppelverbindung aus alkoholischer Lösung in flachen Tafeln und gelben sänlenförmigen Nadeln krystallisierte. Beide Formen zeigten keinen scharfen Schmelzpunkt: sie wurden gegen $80^{\circ}$ wachsartig weich and schmolzen bei $128-130^{\circ}$ zu trüben, gegen $136^{\circ} \mathrm{klar}$ werdenden Flüssigkeiten. (Das aus Brompropiophenon durch Einwirkung von Methylamin gewonnene Produkt lieferte als Golddoppelsalz gelbe Nadelbüschel vom F. $120^{\circ}[\mathrm{l}$. c.].)
$0.3468 \mathrm{~g}$ des exsikkatortrockenen Salzes lieforten $0.1362 \mathrm{~g}$ Cold. Berechnet für $\mathrm{C}_{10} \mathrm{H}_{13} \mathrm{NO} . \mathrm{HAll}_{4}$ : Cefunden: Au 30,19 $39,27 \%$

Der Wert dieses auf das Goldsalz des Methylamidoketons hinweisendeu Analysonresultates wird indessen dadurch stark in Frage gestellt, daß ein aus doni Filtrat der Goldbestimmung dargestelltes Platindoppelsalz, dessen rote siiulenförmige Nadeln bei $182^{\circ}$ unter Aufschäumen schmolzen, einen wesentlich zu hohen Platingehalt $(28,78$ statt $27,50 \%)$ aufwies. Ebenso lieb sich aus dem Filtrat dieser Goldbestimmung und den von Platin durch Schwefelwasserstoff befreiten Mutterlangen nur ein Quecksilberdoppelsalz in Form kleiner weilber Nadeh vom F. $163^{\circ}$ gewinnen. das mit der bei $165^{\circ}$ schmelzenden Doppelverbindung des Amidoketons übereinstimmte.

$0.2379 \mathrm{~g}$ des exsikkatortrockenen salzes lieferten $0,1212 \mathrm{~g}$ figs.

Bereclinet für $\left({ }_{9} \mathrm{H}_{11} \mathrm{NO} . \mathrm{H}\left(\mathrm{l} l . \mathrm{HgCl}_{2} . \mathrm{H}_{2} \mathrm{O}\right)\right.$ : Gefunden: Hig 43,50 $43,93 \%$

Der Versuch hatte somit nicht zu einer betainartigen Verbindung geführt. Ebensowenig gelang es, eine derartigcVerbindung durch Erhitzen des salzsauren Amidoketons mit überschïssigem Dimethylsulfat auf dem Wasserbad am Rückflußkühler darzustellen. Lediglich das Ausgangsmaterial konnte hierbei zurückgewomnen werden.

Völlig resultatlos verlief auch ein Versuch, Dimethylsulfat auf das Amidoketon in Benzollösung einwirlien zu lassen. Anch hierbei resultierte nur unverändertes Ausgangsmaterial und, da die Benzollösung die Anwendung der freeen Base erforderlich machte. das im nachstehenden gekennzeichnete Dimethyldiphenylpyrazin 
rom F. 121-1230, welches durch Ueborfühıng in das bei $247^{\circ}$ schmelzende Silbersalz charakterisiert werden konnte.

Diese Versuche bestätigen somit, daß die Anwesenheit ciner Alkoholgruppe eine der Grundbedingungen für die Bildung der betainartigen Verbindung darstellt.

Trotzdem gelang es nicht, durch halbstiundiges Erhitzen von I is salzsauren Ephedrins mit $\tilde{o} \mathrm{~g}$ Dimethylsulfat am Rückflußkühler auf dem Wasserbad das ron $\mathrm{E}$. S $\mathrm{Se}$ h $\mathrm{m}$ i d t (l. c.) isolier te betainartige Ephedrinderivat zu erhalten. Das erst bei dem Durchschütteln des Reaktionsgemisches mit Aether sich abscheidende Reaktionsprodukt krystallisiente aus Alkohol in Form feinverzwejgter Nadeln vom F. $130^{\circ}$ und erwies sich als schwefelsaures Ephedrin, bei dem cigentümlicherweise auf I Mol. Schwefelsäuc nur I Mol. der Base zil kommen scheint.

$0.2073 \mathrm{~g}$ des exsiklkatortrockenen Salzes ergo ben $0.1798 \mathrm{~g} \mathrm{BaSO}$.

Berechnet für $\mathrm{C}_{10} \mathrm{H}_{15} \mathrm{NO} . \mathrm{H}_{2} \mathrm{SO}_{4}$ :

$$
\text { S } 12,18
$$

Gefunden:

$11,91 \%$

Aus dem Filtrat dieser Bestimmung lieb sich unverändertes Ephedrin vom F. $38^{\circ}$ zurückgewinnen.

\section{Versuch einer Finwirkung von Methylalkohol auf salzsaures Amido- äthylphenylcarbinol.}

Um zu einer Verbindung der Zusammensetzung $\mathrm{C}_{10} \mathrm{H}_{15} \mathrm{NO}$ zu gelangen, wurde nunmehr versucht, mit berechneten Mengen Methylalkohol auf das Amidocarbinol einzuwjken, ähnlich wie Anilin durch Methylalkohol je nach Wunsch in Mono- bzw. Dimethylanilin übergeführt werden kann. Zu diesem Zweck wurde je 1 g des salzsauren Amidocarbinols mic der einem bzw. zwei Mol. entsprechenden Menge Methylalkohol im Rohr 5 Stunden anf 170 - $80^{\circ}$ erhitzt. Der Inhalt der druckfreien Röhren bestand aus einer dunkelbraunen fesien Masse neben einem braunen Liqujdum. Durch Umkrystallisieren des mit Aether und Aceton vorbehandelten Rohrinhaltes aus Alkohol und verdünnter Salzsäure komte indessen nur Ausgangsmaterial wiedergewonmen werlen, das durch seinen F. $188^{\circ}$ bzw. $190^{\circ}$ sowie durch Bestimmung des Chlorgehaltes identifiziert wurde.

$0,2150 \mathrm{~g}$ verbrauchtej $11.50 \mathrm{ccm} 1: 10^{-\mathrm{N}}$-Silbernitratlösming = $0.0408 \mathrm{~g}$ (t)
Berechnet fuir $\left.\left.\mathrm{C}_{9} \mathrm{H}_{13} \mathrm{NO}.\right]_{7} \mathrm{C}\right]$ :
(1) 18,90
Gefunden:
$18,97 \%$

Beide Versuche hatten somit ebensowenig wie die vorhergelienden zu einem monomethylierten Produkt geführt.

\section{Einwirkung von Jodmethyl auf freies Amidoäthylphenylketon.}

Die Versuche, wolche ich weiter anstellte, um durch Einwirkung von Jodmethyl auf Amidoäthylphenylketon ein Monomethylamidoketon zu erhalten, scheiterten an der Unbestindigkeit des freien Amidopropiophenons, das schon nach kurzer Zeit in ein Pyrazinderivat, das Diphenyldimethylpyrazin, übergeht. Da jedoch immerhin die Möglichkeit bestand, dalß die Einwirkung des Jodmethyls eher erfolgte als die Kondensation zum Pyrazin, wurde 
die methylalkoholische Lösung des salzsauren Amidoäthylphenylketons mit Jodmethyl und darauf mit berechneten Mengen NormalKalilauge versetzt. Indessen erwies sich hierbei die Pyrazinkondensation als die glattere Reaktion. Dementsprechend resultierte in der Hauptsache nur Dimethyldiphenylpyrazin: $\mathrm{C}_{18} \mathrm{H}_{10} \mathrm{~N}_{2}$, in Gestalt farbloser glänzender Nadelgruppen vom F. 1250, die im Exsikkator undurchsichtig wurden und sich in allen Eigenschaften als identiseh mit der von $\mathrm{Chr}$. S $\mathrm{ch} \mathrm{mid} \mathrm{t}^{\mathrm{I}}$ ) durch Einwirkung von alkoholischem Ammoniak auf Propiophenonamin erhaltenen Verbindung nachstehender Zusammensetzung erwiesen.

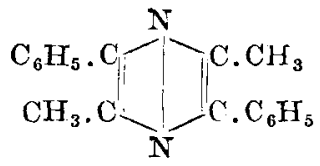

Dieselbe Verbindung ist von $\mathrm{Kol} \mathrm{b}^{2}$ ) auch aus Bromphenylaceton und alkoholischem Ammonjak sowie aus dem durch Reduktion des Isonitrosophenylacetons gewonnenen Amidophenylaceton durch Zusammenbrinegn mit Natronlauge dargestellt worden. Eine weitere, die Angaben $\mathrm{B}$ ehr-Bregow sk i's ${ }^{3}$ ) und $\mathbf{K}$ olb's berichtigende Arbeit $\mathrm{G}$ a brie l's ${ }^{4}$ ) ist mir erst nach AbschluB meiner Arbeit bekannt geworden. Identifiziert wurde dic Verbindung durch Elementaranalyse sowie durch Ueberführung in verschiedene Salze bzw. Doppelsalze.

1. $0.2133 \mathrm{~g}$ lieferten $0.6455 \mathrm{~g} \mathrm{CO}$ und $0.1222 \mathrm{~g} \mathrm{H}_{2} \mathrm{O}$.

2. $0,2139 \mathrm{~g}$ lieferten $0,0.440 \mathrm{~g}\left(\mathrm{O}_{2}\right.$ und $0,1220 \mathrm{~g} \mathrm{H}_{2} \mathrm{O}$.

3. $0.1872 \mathrm{~g}$ verbrauchten nach $\mathrm{K} j$ e l d a h l $13.89 \mathrm{cem} 1 / 10^{-\mathrm{X}}$ ficl entsprechend $0,01945 \mathrm{~g} \mathrm{~N}$.

Berechmet für $\left({ }_{18} \mathrm{H}_{16} \mathrm{~N}_{2}\right.$ :

$$
\begin{array}{rr}
\text { C } & 82.77 \\
\text { It } & \mathbf{6 . 3 0} \\
\mathrm{N} & 10.94
\end{array}
$$

Gefunden :

$\begin{array}{cc}82.53 & 2.82 .11 \% \\ 6,41 & 6,38 \% \\ 3.10,39 \% & \end{array}$

Das nur aus konzentrierter Salzsïure in farblosen Nadeln krystallisierende Hydrochlorid schmolz sofort nach der Isolierung (durch Abpressen zwischen Filirierpapier und zweistündiges Aufbewahren an der Luft getrocknet) erstmalig bei $95^{\circ}$, nahm dann gegen $105-110^{\circ}$ wieder feste Form an und schmolz erneut be157-1590. Schon durch 24stündiges Aufbewahren über Aetzkalk verlor es seinen Chlorgehalt bis auf einen kleinen Rest, so daß der Schmelzpunkt auf $124^{\circ}$ sank. Ein nach 24stündigem Liegen an der Luft analysiertes Hydrochlorid ergab folgende Werte:
1. $0.3666 \mathrm{~g}$ verbrauchten
a) $17,92 \mathrm{ccm} 1 / 10^{-\mathrm{N}}-\mathrm{NaOH}$,
b) $18,03 \mathrm{ccm} 1 / 10^{-\mathrm{N}}$ - $\mathrm{AgNO}$
2. $0,0808 \mathrm{~g}$ verbrauchten
a) $3.99 \mathrm{ccm} 1 / 10^{-\mathrm{N}}$. $\mathrm{NaOH}$.
b) $4,07 \mathrm{ccm} \mathrm{1/10-N.-AgiO_{3 }}$.

\footnotetext{
1) Berichte 2: (1889), 3253.

2) Annalen 291, 267.

3) Berichte 30 (I897), $1522 \mathrm{ff}$.

4) Berichte 41 (1908), 1127.
} 
Berechnet für:
$\mathrm{C}_{18} \mathrm{H}_{16} \mathrm{~N}_{2}$. $\mathrm{HCl}$
$\mathrm{C}_{18} \mathrm{H}_{16} \mathrm{~N}_{2} .2 \mathrm{HCl}$
21,16
1. a) 17,33
2. a) 17,51
Cl 12,12
b) $17.44 \%$

Gefunden:

Nach 70tägigem Aufbewahren des Salzes zunächst über Aetzkalk, dann über $\mathrm{H}_{2} \mathrm{SO}_{4}$ betrug der anfangs rapide Gewichtsverlust $31,18 \%$, der Chlorgehalt $0,47 \% \mathrm{Cl}$.

Das ebenfalls nur aus konzentrierter Salzsäure durch Zusatz von Platinchlorid einheitlich erhaltene Platindoppelsalz bildete feine orangefarbene Nadelbüschel.

1. $0,161 \mathrm{l} \mathrm{g}$ (bei $100^{\circ}$ getrocknet) ergaben $0,0340 \mathrm{~g}$ Platin.

2. $0,4495 \mathrm{~g}$ (bei $100^{\circ}$ getrocknet) ergaben $0.0945 \mathrm{~g}$ Platin.

3. $0,1024 \mathrm{~g}$ (bei $100^{\circ}$ getrocknet) orgaben $0,0220 \mathrm{~g}$ Platin.

Berechnet fiir $\left(\mathrm{C}_{18} \mathrm{H}_{16} \mathrm{~N}_{2}\right)_{2} \mathrm{H}_{2} \mathrm{PtCl}_{6}$ :

$$
\text { Pt } 20,89 \begin{array}{lll}
1.21,11 & 2.21,02 & 3.21,48 \%
\end{array}
$$

In der Literatur noch nicht beschrieben sind die aus konzentrierter salzsaurer Lösung dargestellten Doppelsalze des Quecksilbers und des Goldes.

Das Quecksilbersalz, bildete Tafeln und flache Nadeln, die nach Erweichen (bei $180^{\circ}$ ) zwischen 193 und $195^{\circ}$ schmolzen.

$0,2085 \mathrm{~g}$ des exsilskatortrockenen Salzes ergaben $0,0525 \mathrm{~g} \mathrm{HgS}$.

Berechnet für $\left(\mathrm{C}_{18} \mathrm{H}_{16} \mathrm{~N}_{2} .2 \mathrm{HCl}\right)_{2} \mathrm{HgCl}_{2}$;

$$
\text { Hig } 21,39
$$

Gefunden :

$$
21,70 \%
$$

Die Golddoppelverbindung schied sich aus salzsaurer Lösung in hellgelben Flocken, aus alkoholhaltiger in gelben durchsichtigen Blättchen vom F. $115^{0}$ aus.

1. $0,3525 \mathrm{~g}$ verloren bei $100^{\circ} 0,0093 \mathrm{~g}$; der Rückstand $(0,3432 \mathrm{~g})$ ergab $0,1124 \mathrm{~g} \mathrm{Au}$.

2. $0.2172 \mathrm{~g}$ verloren bei $100^{\circ} 0,0060 \mathrm{~g}$; der Rückstand $(0,2112 \mathrm{~g})$ ergab $0,0691 \mathrm{~g} \mathrm{Au}$.

$$
\begin{array}{cc}
\text { Berechnet fïr }\left(\mathrm{C}_{18} \mathrm{H}_{16} \mathrm{~N}_{2} . \mathrm{HAuCl}_{4}+\mathrm{H}_{2} \mathrm{O}\right): & \text { Gefunden: } \\
\mathrm{H}_{2} \mathrm{O} 2,9 \mathrm{1} & \begin{array}{l}
2.64,2.2,76 \% \\
\text { Berechnet für }\left(\mathrm{C}_{18} \mathrm{H}_{16} \mathrm{~N}_{2} . \mathrm{HAuCl}_{4}\right):
\end{array} \\
\mathrm{Au} 32,87 & \text { Gefunden: } \\
& \text { 1. } 32,75,2.32,72 \%
\end{array}
$$

Nach Zusatz von Silbernitrat zu einer heißen alkoholischen Lösung des Pyrazinderivates resultierte mit und ohne Salpetersäurezusatz nach wenigen Sekunden ein Silbernitratdoppelsalz in Gestalt feiner glänzender Nadeln vom F. 2470 (unter Aufschäumen). Der schwankende Trockenverlust $(3,4-5,75 \%)$ des durch Wasser vollkommen zerfallenden Salzes ließ die Aufstellung einer Formel für das ungetrocknete Salz nicht zu. Dagegen scheint der bei $100^{\circ}$ getrockneten Substanz die Zusammensetzung $\mathrm{C}_{18} \mathrm{H}_{16} \mathrm{~N}_{2} \cdot \mathrm{AgNO}_{3}$ zuzukommen. Der Silbergehalt von 5 Analysen betrug im Mitiel $25,19 \%$, berechnet für obige Formel $25,08 \%$ Ag.

Neben diesem Pyrazin resultierten aus den Mutterlaugen noch ein bei $126^{\circ}$ schmelzendes Jodid sowie, nach dem Umsetzen mit Chlorsilber, ein Chlorid vom F. $175^{\circ}$, deren Halogengehalt jedoch nicht dem gesuchten Produkt entsprach, und unverändertes Ausgangsmaterial.

Das Ergebnis der bisherigen Versuche lehrt somit, daß die direkte Methylierung des Amidoäthylphenylketons bzw, -carbinols mit den gebräuchlichen Hilfsmitteh nicht $n$ der gewïnschten 
Verbindung führt. Mit Rücksicht anf diese Erfahrung wurde nunmehr die Darstellung des gesuchten Produktes auf dem Lmweg über das Acetyl- bzw. Benzoylcarbinol angestrebt.

\section{Methylierungsversuche mit acetyliertem bzw. benzoyliertem Amido- arbinol.}

A cetylierung des tomidoäth $y^{3}$ phenylearbinols. Zum Zweek der Acetylierung wurde $1 \mathrm{~g}$ des salzsanren Amidocarbinols mit der 10-fachen Menge Essigsäureanhydrid am Rückflußkühler im schwachen Sieden erhalten, bis nach erfolgter Lösung eine Verfärbung der Flüssigkeit eingetreten war (ein längeres $\mathrm{Er}$ hitzen führte zu öligen Produkten). Nach achttägigem Stehen des durch Einengen mit Alkohol vom Anhydridüberschuß befreiten Rückstandes im Exsikkator bilde'en sich weiße sejdenglänzende Nadelbüschel, die sich als halogenfrei erwiesen und bei $78^{\circ}$ schmolzen. Da diese Verbindung weder mit Gold-noch mit Platinchlorid krystallisierbare Doppelverbindungen lieferte, wurde von einer analy ischen Charakterisierung Abstand genommen. Dabei muß allerdings die Frage offen bleiben, ob ein N-Acetylderivat vorlag, oder ob die Acetylgruppe am Sauerstoff angetreten war. Im letzteren Fall hätten die basischen Eigenschaften erhalten bleiben müssen, während die Acidyliernng am stickstoff dic Basizität schwächte.

Versuche zur Methylierung dieses Acetylderivates mit .Jodmethyl verliefen ergebnislos. Werler durch mehrwöchiges Stehen bei gewöhnlicher Temperatur, noch durch zweistündiges Erhitzen am Rückflußkühler, noch auch dureh zwölfstündige Einwirkung ron Jodmethyl im Einsehmelzrohr bei $100^{\circ}$ ließen sich jodhaltige $A$,bscheidungen gewinnen, so daB es den Anschein hat, als ob clurch Einführung der Acetylgruppe die Substitution des restierenden Amidowassersizoffatoms verhindert wird.

Ebenso resultatlos verliefon die gleichzeitig angestellten Versuche zur Methylierung der Benzoylderivate des Amidoäthylphenylcarbinols. Ueber die Darstellung der beiden Benzoylverbindungen habe ich bereiss früher ${ }^{3}$ ) berjohtet. Analog rem passiven Verhalien des Acecylproduktes resultierten auch hier bei sechsstündigem Erhitzen der Mono- bzw. Dibenzoylverbindung mit Jodmethyl im Einselı melzrohr auf $100^{\circ}$ keinerlei jodwasserstoffsaure Salze. Jeeliglich der Schmelzpunkt des Dibenzoylamidocarbinols (bisher 1620) erfuhr durch diese Behandlungsweise eine Erhöhung auf $165^{\circ}$. Dagegen wurde die Beobachtung gemacht, daß sich diese Verbindung durch Behandeln mit Salzsaiure und Alkohol in ein nach vorhergehendem Sintern bei $263^{\circ}$ selmelzendes Hydrochlorid überführen läBt, das aus alkoholischer Lösung in Form stark verästelter Nadeln krystallisiert.

$0,1891 \mathrm{~g}$ verbrauditen $4.80 \mathrm{ccm} 1 / 10 \mathrm{~N}$.-Silhernitrat.

Berechnet fiir $\mathrm{C}_{9} \mathrm{H}_{11} \mathrm{NO}\left(\mathrm{C}_{6} \mathrm{H}_{5}, \mathrm{CO}\right)_{2} . \mathrm{HCl}$ : Gefunden :

$$
\text { C1 } 8.98 \quad 9,01 \%
$$

Diese Beobachtung wirkt um so befremdender, als dic Methylierungsversuche gezeigt hatten, daß die basischen Eigenschaften

1) Arch. d. Pharm. 255 (1917) 147. 
der Amidoverbindungen, wenigstens gegenüber Jodmethyl, schon durch den Eintritt nur einer Acylgruppe verdeckt werden, während das Hydrochlorid eine durchaus beständige Verbindung darstellt, die ihren Chlorgehalt selbst bei einjährigem Aufbewahren nicht verloren hatte.

Die im rorstehenden beschriebenen Versuche hatten somit die Unbrauchbarkeit auch dieser Methode für die beabsichtigte Methylierung ergeben.

\section{Alkylierungsversuche mit Aldehyden.}

Im Hinblick auf die bisherigen negativen Resultate wurde nunmehr versucht, durch Einwirkung von Formaldehyd direkt zu ejnem Methyl- oder doch zu einem Methylenderivat des Amidocarbinols zu gelangen. Andererseits sollte versucht werden, durch Umsetzung mit Benzaldehyd entweder eine Benzyl- oder eine Benzalverbindung $z u$ erhalten und das gesuchte Methylierungsprodukt dann auf dem Umweg über eine quartäre Verbindung durch Wjederabspaltung des Benzaldehyds zu gewinnen (s. u.).

\section{Methylierungsversuche mit Formaldehyd.?}

Entsprechend der E s c h w e i 1 e r $)^{1}$ schen Gleichung $2\left[-\mathrm{NH}_{2}\right]$ $+3 \mathrm{CH}_{2} \mathrm{O}=2\left[-\mathrm{NHCH}_{3}\right]+\mathrm{CO}_{2}+\mathrm{H}_{2} \mathrm{O}$ wurde eine konzentrierte wässerige Lösung von $1 \mathrm{~g}$ des salzsauren Amidocarbinols $(=0,813 \mathrm{~g}$ freier Base) mit $0,65 \mathrm{~g}$ einer $38 \%$ igen Formaldehydlösung im Bombenrohr 4 Stunden lang auf $130^{\circ}$ erhitzt. Nach Behandlung des teilweise braungefürbten Rohrinhaltes mit heißem Wasser (zur Zersetzung einer etwa gebildeten Methylenverbindung und zur Entfermung harzartiger Reaktionsprodukte) und verceblichen Krystalljsationsversuchen wurde der Verdunstungsrückstand der wässerigen Lösung durch Darstellung eines ölig bleibenden Quecksilberdoppelsalzes vorgereinigt und aus diesem dann, nach Intfernung des Metalls, eine Pláfindoppelverbindung in rotgelben körnigen K̇rusten vom F. $171^{\circ}$ gewonnen. Die Ausbeute war jedoch so gering und die Krystallabscheidung so wenig charakteristisch, da $\beta$ von der Wiederholung des Versuches mit größeren Materialmengen abgesehen wurde.

Ebenso ergebnislos verlief der Versuch, unter Anwendung der freien Base 2 Mol. Formaldehyd auf 1 Mol. der Amidorerbinchung, entsprechend den Angaben von $\mathrm{H}$ e s s und $\mathrm{U}$ i b r i g ${ }^{2}$ ), einwirken zu lassen. Nach fünfstündigem Erhitzen in Einschmelzrohr auf $100^{\circ}$ ließen sich aus dem Rohrinhalt nur minimale Mengen zweier Quecksilberdoppelsalze darstellen. Die zuerst isolierten farblosen Tafeln schmolzen bei 95 bis $97^{\circ}$, weitere Krystallabscheidungen in Form warziger Krusten zeigten den F. $160^{\circ}$.

Eine Einwirkung des Formaldehyds auf das Amidocarbinol war somit nicht in der erwünschten Weise erfolgt.

\section{Reaktionsversuche mit Benzaldehyd.}

In Anlehnung an die Versuche von $H$ ess und Uibrig (l. c.) wurde $1 \mathrm{~g}$ des salzsauren Amidopropiophenons mit $1 \mathrm{~g}$ Benz-

1) Berichte 38 (1905), 880.

2) Berichte 48 (1915). 1974. 
aldehyd und $4 \mathrm{~g}$ Alkohol bei Gegenwart konzentrierter Salzsäure 7 Stunden lang im Wasserbad auf $90^{\circ}$ erwärmt. Das im Vakuum eingeengte und durch Extraktion mit Aether vom Benzaldehydüberschuß befreite Reaktionsprodukt bestand aus schwach bräunlichen Krystallflittern vom F. $174^{\circ}$, die sich in heißem Aceton, leicht auch in Wasser und Alkohol lösten und mit Goldchlorid ein öliges Doppelsalz lieferten. Die Platindoppelverbindung dieses Salzes bildete rotgelbe durchsichtige Nadeln, die unter Aufschäumen bei $187^{\circ}$ schmolzen, deren Platingehalt indessen sogar den für die Doppelverbindung desAusgangsmaterials berechneten Wert überstieg.

\section{$0,2770 \mathrm{~g}$ ergaben $0,0794 \mathrm{~g}$ Platin. \\ Berechnet fïr $\left(\mathrm{C}_{9} \mathrm{H}_{11} \mathrm{NO}\right)_{2} \mathrm{H}_{2} \mathrm{PtCl}_{6}$ : Pt 27.55 \\ Gefunden : $28,66 \%$}

Das gesuchte Reaktionsprodukt war somit unter obigen Bedingungen nicht erhalten worden.

Gleichzeitig wurde die Darsiellung eines Einwirkungsproduktes von Benzaldehyd auf treies Amidoäthylphe yyl arbinol in Angriff genommen. $1 \mathrm{~g}$ des Amidocarbinols wurde nit $1,5 \mathrm{~g}$ Benzaldehyd und wenig Aether 7 Stunden lang am Rïickflußkühler erhitzt. Da der ölige Verdunstungsrückstand des Reaktionsgemisehes nicht krystallinis ch erhalten werden konnte, andererseits aber eine Probe beim Aussehütteln mit 10\%iger Salzsüure die farblosen Krystalltafelı des Ausgangsmaterials (F. 187-1890) lieferte, wurde angenommen, daß eine Verbindung des Benzaldehyds mit Amidocarbinol vorlag, und daß diese durch die Säurebehandlung eine Spaliung erfahren habe. Unter Berücksichtigung dor mangelnden Krystallisierbarkeit des Resktionsproduktes wurde die Hauptmenge direkt der Me hylierung unterworfen und zu diesern Zweck mit Jodmethyl mehrere Tage bei Zimmertemperatur in Reaktion belassen; dem braunen öligen Verclunstungsrückstand wurden durch Ausschütteln mit Wasser die löslichen Anteile entzogen.

Neben geringen Mengen jodfreier Nadeln vom F. $118^{\circ}$ (Benzoe-

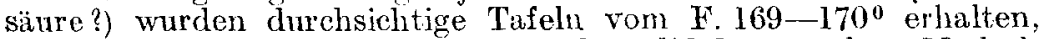
die ihrem Jodgehalt nach aus dem Hydrojodid des gesuchten Methylamidoäthylphenylearbinols zu bestehen schienen, mithin keine Benzoylgruppe enthielten.

0.1711 g des bei $100^{\circ}$ getrockneten Hydrojodids verlorauchten $5.90 \mathrm{ccm} 1 / 10^{-\hat{N}}$.-Silberlösung und lieferten $0,1389 \mathrm{~g} \mathrm{Ag}$.

$$
\text { Bereclmet für } \mathrm{C}_{10} \mathrm{H}_{15} \mathrm{NO} . \mathrm{HJ}: \quad \begin{gathered}
\text { Gefunden : } \\
43,86 \%
\end{gathered}
$$

Téür die Richtigkeit dieser Annahme spricht ferner die Form der aus dem Filtrat dieser Bestimmung dargestellten freien Base, die sich als weißer, aus seidenglänzenden Nadeln bestehender Wandbelag abschied. Das zur weiteren Charakterisierung dargestellte Golddoppelsalz bildetc hellgelbe flache Nadeln, die (nach Erweichen bei $108^{\circ}$ ) bei $111,5^{\circ}$ schmolzen und somit den Eigenschaften des durch Reduktion des Methylamidopropiophenons erhaltenen Doppelsalzes (F. 1150) nahekommen.

Da ein unter gleichen Bedingungen, aber ohne Benzaldehyd, angestellter Versuch nur ein quartäres Jodid li fe it' ${ }^{\prime}$ : so dürfte 
A. Eberhard: Inaktives Ephedrin bez. Pseudoephedrin. 113

die Entstehung der in etwas größeren Mengen als bisher isolierten Methylamidoverbindung nur auf die intermediäre Bildung einer Benzal- oder einer von dieser abgeleiteten quartären Verbindung im Sinne der von $\mathrm{Decker}$ und $\mathrm{B} \mathrm{e} \mathrm{ck} \mathrm{er}{ }^{1}$ ) angegebenen Darstellungsweise sekundärer Amine zurückzuführen sein. Wie diese Autoren nachgewiesen haben, entsteht durch Kondensation primärer Amine mit einem Aldehyd ein Alkylidenamin, das durch Addition von Jodmethyl eine quartäre Verbindung liefert. Diese erfährt dann durch Hydrolyse eine Spaltung in Aldehyd und ein sekundäres Amin mit der Alkylgruppe des Jodalkyls im Sinne der Gleichungen:

$$
\begin{gathered}
\mathrm{R} \cdot \mathrm{NH}_{2}+\mathrm{R}^{\prime \prime} \cdot \mathrm{CHO} \rightarrow \mathrm{R}^{\prime \prime} \cdot \mathrm{CH}=\mathrm{NR}+\mathrm{H}_{2} \mathrm{O} \\
\mathrm{R}^{\prime \prime} \cdot \mathrm{CH}=\mathrm{NR}+\mathrm{R}^{\prime} \mathrm{J}=\mathrm{R}^{\prime \prime} \cdot \mathrm{CH}=\mathrm{N} \mathrm{N}_{\mathrm{J}}^{-\mathrm{R}^{\prime}} \\
\mathrm{R}^{\prime \prime} \cdot \mathrm{CH}=\mathrm{N} \mathrm{N}_{\mathrm{J}}^{\mathrm{R}^{\prime}}+\mathrm{H}_{2} \mathrm{O}=\mathrm{R}^{\prime \prime} \cdot \mathrm{CHO}+\mathrm{N}>_{\mathrm{H}}^{-\mathrm{R}^{\prime}}+\mathrm{HJ} .
\end{gathered}
$$

Da weitere Versuche, auf diese Weise die Zwischenprodukte oder größere Mengen des Methylierungsproduktes zu erhalten, nicht zum Ziel führten, wurden die Versuche, auf dem Umweg über das Amidocarbinol $\mathrm{zu}$ ephedrinähnlichen Verbindungen $\mathrm{zu}$ gelangen, vorläufig aufgegeben.

Alle bisherigen Methylierungsversuche des Amidopropiophenons auf direktem und indirektem Wege haben somit entweder völlig versagt oder doch nur so geringe Ausbeuten geliefert, daß eine Charakterisierung des Reaktionsproduktes meist unmöglich war. Im allgemeinen haben die vorstehenden Versuche gezeigt, dab die Anwendung berechneter Mengen der Methylierungsmittel nicht zum Ziel führen kann, da die Reaktion bis zur Bildung quartärer Verbindungen fortschreitet. Weiter ergab sich, daß die Methylierung der salzsauren Salze wesentlich größere Schwierjgkeiten bietet als die der freien Amidobase, und daß Methylierungsversuche des freien Amidopropiophenons, infolge der eintretenden Pyrazinbildung, von vornherein als erfolglos erscheinen müssen. Die beste Ausbeute an dem gesuchten Methylamidoäthylphenylearbinol lieferte noch der unter Anwendung von Benzaldehyd und Jodmethyl vorgenommene Versuch; inclessen lehrt die auch in diesem Fall nur geringe Ausbente, daß die Hauptreakiion nicht in dem gewünschten Sinne verläuft. Da die Resultate, welche bei der Einwirkung von Methylamin anf Brompropiophenon erzielt wurden, wesentlich besser waren als die, welche nach den vorstehenden Angaben erzielt wurden, so gelangte die Darsiellung des gesuchteu Produktes nummehr nur auf dem Wege über das Brompropiophenon zur Ausfühu'ung.

1) Annalen 395 (1913), 362. 
114 A. Eberhard: Inaktives Ephedrin bez. Pseudoephedrin.

\section{Synthese aus Methylamin und Brompropiophenon. $a$-Methylamido-Aethylphenylketon.}

Die Darstellung des $\alpha$-Methylamido-Aethylphenylketons erfolgte im wesentlichen nach don früher gemachten Erfahrungen im Sinne der Gleichung:

$$
\mathrm{C}_{6} \mathrm{H}_{5} \cdot \mathrm{CO} \cdot \mathrm{CHBr} \cdot \mathrm{CH}_{3}+\underset{\mathrm{NH}_{2} \mathrm{CH}_{3} \cdot \mathrm{HBr}_{3}}{2 \mathrm{~N} \mathrm{H}_{3}} \mathrm{CH}_{3}=\mathrm{C}_{6} \mathrm{H}_{5} \mathrm{CO} \cdot \mathrm{CH}\left(\mathrm{NHCH}_{3}\right) \cdot \mathrm{CH}_{3}+
$$

Mit Rücksicht auf die geringe Löslichleit des Methylamins in Benzol und die dadurch erforderliche Verarbeitung relativ großer Flüssigkeitsmengen wurde von der Verwendung benzolischer Lösungen abgesehen und statt dessen in absolut-alkoholischer Lüsung goar beitet. Vorversuche mit kleinen Mengen bestätigten die bereits früher gemachte Beobachtung, dals die Einwirkung von Methylamin auf Brompropiophenon in der Kälte nur langsam erfolgt und daß dementsprechend rlie Menge des unveränderten Materials bei längerem Stehen des Reaktionsgemisches abnahm. Allerdings ging damit eine stärkere Braunfürbung des Reakioionsproduktes parallel. Bei der Verwendung von 2 Molekülen $\mathrm{NH}_{2} \mathrm{CH}_{3}$ auf 1 Molekül Brompropiophenon ergab sich eine starke Verunreinigung der gesuchten $\checkmark$ rerbindung durch Methylaminsalze, indessen muBte dieser Uebelstand in Kauf genommen werden, da ein mit äquimolekularen Mengen der Reagentien ausgeführter Versuch nur eine wesentlich geringere Ausbeute lieferte. Win weiterer Versuch, das Methylamidoketon durch Einleiten gasförmigen Methylamins in äther ische Brompropiophenonlösung zu gewinnen, zeigte gegenüber der bisherigen Darstellungsmethode keine Vorteile, da das Verfahren infolge der hohen Lioslichkeit des Methylamins eine ständige sorgfältige Ueberwachung erforderte, ohne da $B$ die Ausbeute besonders gut gewesen wäre. Dureh Ueberführung in das Hydrochlorid und Umkrystallisieren aus heißem Aceton ließ sich eine teilweise Reinigung des Rohproduktes erzielen. Das hierans dargestellte Platindoppelsalz zeigte (allerdings nur in den ersien Krystallabscheidungen), übereinstimmend mit früheren Augaben, den . F. $187^{\circ}$ unter Schwärzung und Aufschäumen. Fine Pt-Bestimmnng bewies die Identität des Reaktionsproduktes mit dem gesuchten Methylamidoäthylphenylketon:

$0,6687 \mathrm{~g}$ des boi $100^{\circ}$ getrockneten Platindoppelsalzes ergaben 0.1776 g Platin.
Berechnet für $\left(\mathrm{C}_{6} \mathrm{H}_{5}, \mathrm{CO} . \mathrm{CH}\left(\mathrm{NHCH} \mathrm{H}_{3}\right) \cdot\left(\mathrm{CH}_{3}\right)_{2} \mathrm{Hi}_{2} \mathrm{PtC} \mathrm{l}_{6}\right.$ : l't 26,52
G $:$ funden : $26,56 \%$

Entsprechend diesen Vorversuchen wurden die unter Eiskühlung portionsweise zusammengebrachten L̈̈sungen von Methylamin ind Brompropiophenon nach vierstündigem Stehen in der Kälte allmählich unter weiterer Kühlung mit verdünnter Salzsäure übersättigt. Die zur Rü «gewinnung des nnverändert gebliebenen Brompropiophenons nunmehr auf ein Drittel ihres Volumens cingeengte und mit Wasser verdünnte Lösung schied nach mehrstündigem Stehen ölige Massen ab, die getrennt und bei einer erneuten Darstellung verwendet wurden. Aus der übers'ehenden Lisung resultierte nach dem Eindunsten und Aufbewahren im 
A. Eberhard: Inaktives Ephedrin bez. Peudoephedrin. 115

Vakuum-Exsikkator ein schwarzbraunes dick-öliges Liquidum, das mit heißem Aceton ausgezogen wurde. Der Verdunstungsrückstand aler Acetonlösung bestand aus einer ebenso dunklen öligen Masse (I).

Der noch immer dunkel gefärbte Rückstand, dem Aceton heine nennenswerten Mengen des Reaktionsproduktes mehr za entziehen vermochte. warde mit kaltem, absolutem Alkohol erschöpft, die eingedampften Auszüge durch Ueberschichten mit Aceton von mitgelösten Methylamjnsalzen möglichst befreit und das erneut eingeengte Fittra durch Sohichten mit Aether gefällt. Das sich am Boden abscheidende dunkelbratme Liquidum (II) zejgte im wesentlichen die Eigenschaften des Verdunstumgsrückstandes der Acetoniösung (I). Die beiden Rohprodukìe wurden in derselben Weise getrennt weiterbehandelt.

Durch Aufnehmen der Rückstände (I und II) mit Wasser und Umsetzung der Filtrate mit Chlorsiber resultierten rote, stark grrün fluoreszierende Lösungen, deren Färbung durch Behandlung mit Kohle aufgehellt werden komnte. Die Verdunstungsrückstände dieser Lösungen waren öliger Natur und gingen auch im VakuumExsikkator nicht in den krystallisierten Zustand über. Erst durch Verrühren mit Aether und sofortiges Einsiellen in den VakuumExsikkator erstarten die Rücksiände zu spröden blasigen Massen. die sich in Essigäther leicht, schwer dagegen in sierlendem Ace:on lösten. Da durch Ueberschichten der alkoholischen Lösungen mit Aether nur ein unreines Produkt resultierte, wurden die Räckstände durch Umkrystallisieren aus siedendem Aceton gereinigt. Es resultierten so flockige Gebilde, die ohne weitere Reinigung zwischen 165-1720 schmolzen, und in diesem rohen Zustand direkt zur Reduktion verwendet wurden.

Aus den lęzten Mitterlangen dieser Acetonlösungen resultierien - selbst nach dem Ansäuem mit Salzsäure -- halogenfreie Produkte von IF. 1180, die bisher nicht weiter untersucht wurden, da sie keine basischen Figenschaften besaßen.

Der in Aceton unlösliche Rüeksiand bestand aus salzsaurem Methylamin, wie clurch Bestimmung des Schmelzpunktes (2240) gezejg werden konnte.

\section{a-Methylamidoäthylphonylcarbinol.}

Gelegentlich der früher $(l, c$.$) vorgenommenen Reduktion des$ Methylamidopropiophenons zu dem entsprechenden Nethylamidocarbinol mit Hilfe von Natriumamalgam in schwach saurer Lösung hatie es sich gezeigt, daß trotz Einhaltung der entsprechenden Vorsichtsmaßregeln ein erheblicher Teil des Materials eine tjefergreifonde Zersetzung erleidet, so daß die Ausbente nur minimal war. Eine Möglichkeit, diesen Uebelstand zu umgehen, bot die Anwendung der katalytischen Hydrierung, vorausgesetzt, dab nicht anch hier dic Belastung der Amilogruppe hemmend anf den Vorlanf der Reaktion einwirkte.

Da Vorversuche nach anfänglichen Schwierigkeiten günstig verliefen, wurden nach dem von $\mathrm{M}$ a $\mathrm{n} \mathrm{n}$ i $\mathrm{ch}^{\mathrm{l}}$ ) angegebenen und von mir bercits mit Erfolg zur Reduktion des Amicloäthylphenyl-

1) Arch. d. Pharm. 1.53 (1915), 181. 


\section{A. Eberhard: Inaktives Ephedrin bez. Psendoephedrin.}

ketons ${ }^{1}$ ) angewandten Verfahren je $5 \mathrm{~g}$ des rohen salzsauren Methylamidoäthylphenylketons mit der Hälfte ihrer Menge Palladiumkohle (2\% $\mathrm{Pd}$ enthaltend) und der zur Lösung des Methylamidoketons erforderlichen Menge. Wasser unter einem Ueberdruck von etwa $1 / 3$ Atmosphäre in der ,Schüttelente" der katalytischen $\mathrm{Hy}$ drierung unterworfen. Nach anfänglich nur langsamer Wasserstoffaufnahme stieg diese nach ein bis zwei Stunden auf ihren Höhepunkt, so daß sich im Schüttelgefäß ein luftverdünnter Raum bildete, um dann wieder stark nachzulassen. Die Hydrierung gelang am besten mit reinem Ausgangsmaterial, sie versagte fast vollkommen, sobald größere Mengen salzsauren oder bromwasserstoffsauren Methylamins zugegen waren, z. B. als versucht wurde, das zum Sirup eingedampfte Reaktionsprodukt von Methylamin und Brompropiophenon (s. o.) oder ein ungenügend gereinigtes Material zu hydrieren, um so das lästige Umkrystallisieren aus heißem Aceton zu umgehen und auf diesem Wege das auch aus wässeriger Lösung gut krystallisierende, durch Hydrierung entstehende salzsaure Methylamidoäthylphenylcarbinol von den begleitenden Methylaminsalzen durch Krystallisation zu trennen.

Versuche, die Reaktion unter gewöhnlichem Atmosphärendruck analog dem Verfahren von B u s c h ${ }^{2}$ ) durchzuführen, mißlangen vollständig. Weder bei gewöhnlicher Temperatur noch im Wasserbad konnte durch zweistündiges Einleiten von $\mathrm{H}_{2}$ eine Hydrogenisation hervorgerufen werden.

Dagegen resultierten nach obigem Verfahren nach dem Abfiltrieren der Palladiumkohle und Einengen des Filtrates unter Zusatz von wenig verdünnter Salzsäure farblose, gut ausgebildete rhombische Tafeln von F. $184^{\circ}$. Durch Reinigung über das Platinsalz (s. u.) stieg der F, auf $187^{\circ}$. Diese leichte Krystallisierbarkeit mußte um so mehr befremden, als das seinerzeit durch Reduktion mit Natriumamalgam in saurer Lösung erzielte Hydrochlorid durch besonders leichte Iöslichkeit ausurezeichnet war und selbst aus absolutem Alkohol nicht krystallisiert hatte gewommen werden können. Eine Gewinnung in reiner Form war damals nur durch Behandeln des Verdunstungsrückstandes mit Areton (in Gestalt weißer Warzen vom F. 180/181 ${ }^{\circ}$ und durch Ueberschichten der konzentrierten, absolut alkoholischen Lösung mit Aether (in Form weißer Flocken desselben Schmelzpunktes) möglich gewesen. Da sich auch, abgesehen von der beobachteten $\mathrm{H}_{2}$-Aufnahme bei der Reduktion das Ausgangsmaterial, das salzsaure Methylamidoäthylphenylketon, durch seime leichte Löslichkeit erheblich von dem erhaltenen Reaktionsprodukt untrrscheidet, komnte es sich auch nicht um unverändertes Methylamidoketon handeln. Daß andererseits keine tiefergreifencle Umsetzung bei der Reduktion. eingetreten war, ergab sich zunächst aus cler Bestimımung des Chlorgehalts:

$0.6167 \mathrm{~g}$ des bei $100^{\circ}$ getrockneten Salzes verbrauchten zur Titration $30.27 \mathrm{~g} 1 / 10^{-N}$.-Ag-Lüsung.

Berechnet für $\mathrm{C}_{10} \mathrm{H}_{15} \mathrm{NO} . \mathrm{HCl}$ :

$$
\text { (!) } 17,57
$$

Gefunden : $17,40 \%$

1) Arch. d. Pharm. 255 (1917), 142.

2) Ztschr. f. angew. Chem. 19]8, I., 232. 
Da indessen der Chlorgehalt keinen Aufschluß darüber zu geben vermag, ob wirklich eine Aufnahme von Wasserstoff in dem gewünschten Sinne stattgefunden hat — die Differenz liegt innerhalb der analytischen Fehlergrenzen -, so mußte die Verbindung durch Ueberführung in ihr Acetyl- und Benzoylderivat, in die freie Base und ihre Gold- und Platindoppelsaize näher charakterisiert werden.

\section{Acetylmethylamidoäthylphenylcarbinol.}

Da nach den Angaben Millers (l. e.) Acetylehlorid für die Zwecke der Acetylierung des Ephedrins ungeeignet ist, wurde das von diesem und Calli e B benutzte Essigsäureanhydrid zur Einführung des Säurerestes angewandt.

$1 \mathrm{~g}$ des salzsauren Methylamidocarbinols wurde mit der zehnfachen Menge Essigsäureanhydrid vier Stunden lang am Rückflußkühler im schwachen Sieden erhalten, darauf das überschüssige Anhydrid mit dem zum Nachspülen des Kölbchens benutzten Alkohol auf dem Wasserbad verdampft, und der zähe Rückstand mit Aceton verrieben. Das Aceton nahm nur einen Teil des Verdunstungsrückstandes auf, während der größte Teil des Reaktionsproduktes als weißes, auch in heißem Aceton nur wenig lösliches Pulver zurückblieb. Durch Umkrystallisieren aus Alkohol, besser durch Zusatz von Aceton zur alkoholischen Lösung in der Wärme, resultierten farblose Tafeln, die zwischen 165-1670 schmolzen, Wie durch die Analyse ihres Chlorgehaltes festgestellt werden konnte, handelte es sich um ein Monoacetylderivat der Verbindung $\mathrm{C}_{10} \mathrm{H}_{15} \mathrm{NO}$.

$0,4460 \mathrm{~g}$ der exsiklkatortrockenen Substanz ergaben $0,2658 \mathrm{~g} \mathrm{AgCl}$ Berechnet für $\mathrm{C}_{10} \mathrm{H}_{14}\left(\mathrm{CH}_{3} \mathrm{CO}\right) . \mathrm{NO} . \mathrm{HCl}$ : Gefunden:
Cl 14,56
$14,74 \%$

Diese Verbindung zeigt äußerlich Aehnlichkeit mit dem salzsauren Salz des aus Ephedrin und aus Pseudoephedrin dargestellten Acetylderivates. Auch dieses bildet farblose tafelförmige Krystalle. Der Schmelzpunkt des aus naturellem Material gewonnenen Derivates liegt dagegen $10^{\circ}$ höher (M i lle r, C a 11 i e B).

Zur Darstellung der freien Acetylbase wurde das Filtrat obiger Chlorbestimmung nach der Entsilberung auf ein Drittel seines Volumens eingeengt und nach dem Uebersättigen mit Natronlauge mit Aether ausgeschüttelt. Aus der ätherischen Lösung schieden sich an den Wandungen lange, seidenglänzende Nadeln vom F. $113^{\circ}$ aus. Da es indessen fraglich war, ob nicht durch das Eindampfen der von der Chlorbestimmung herrührenden saueren Lösung eine Abspaltung der Acetylgruppe eingetreten war, wurde eine neue Probe der salzsauren A,cetylverbindung mit Soda und Aether ausgeschüttelt. Die so isolierte freie Acetylbase resultierte indessen nur in Gestalt einer gelblichen (amorphen) dicköligen Flüssigkeit, die weder durch wochenlanges Aufbewahren im Exsikkator noch auch durch Impfen mit kleinen Mengen obiger, bei $113^{\circ}$ schmelzenden Verbindung zur Krystallisation zu bringen war. Ebensowenig gelang es, nach der von $\mathrm{Ca} 1 \mathrm{li}$ e B bei der Acetylverbindung des Ephedrins angewandten Methode, die freie Acetyl-

Axch. d. Pharm. CarviII. Bds. 2. Heft. 
base durch vierstündiges Erhitzen des salzsauren Salzes mit Sorlalösung auf dem Wasserbad und Extrahieren des Reaktionsgemisches mit Aether krystallisiert zu erhalten.

Mithin kann objge, bei $113^{0}$ schmelzende Base nicht aus der Acetylverbindung bestanden haben, vielmehr muß angenommen werden, daß lurch das Einengen in saurer Lösung die Acetylgruppe völlig eliminiert wurde. Diese auskrystallisierte Base muß also ebenfalls als. Methylamidoäthylphenylcarbinol angesprochen werden, wie weiter unten gezeigt wird. Sie simmt im Schmelzpunkt, Gestalt und Gilanz mit der von mir 1914 durch Reduktion des Methylamidopropiophenons mit Natriumamalgam in saurer T_ösung isolierten Base iberein.

\section{Benzoyl-Verbindung $\mathrm{C}_{10} \mathrm{H}_{13}\left(\mathrm{C}_{6} \mathrm{H}_{5} \cdot \mathrm{CO}\right)_{2}$. NO.}

Zur weiteren Kennzeichnung der erhaltenen Verbindung wurde die aus dem salzsauren Salz isolier te freie Base einer Benzoylierung nach Sohot te n-Ba u m a n n unterworfen. Aus der ätherischen Ausschüttelungsflüssigkeit des Reaktionsgemisches resultierte zunächst eine farblose ölige Abscheidung, die allmählich zum Teil in farblose Säulen überging. Durch Behandeln mit Alkohol wurden die öligen Beimengungen entfernt. Die so isolierten Tafeln schmolzen bei $114-115^{\circ}$.

Da infolge der Gaseinsohränkung eine Elementaranalyse nicht durchgeführt werden konnte, mußte die Zahl der eingetretenen Benzoylreste durch Verseifung bestimmt werden, wobei die basische Natır des entbenzoylierten Reaktionsproduktes zu beachten war.

$0.3640 \mathrm{~g}$ des getrockneten Materials verbrauchten scheinbar nur 1.95 acm $1 / 2$-N.-KOHi (Indikator: Methylrot). Aus obiger Menge der Dibmzoylverbindung werden durch die Verseifung $0.1611 \mathrm{~g}$ Methylamidoüthylphenylkarbinol in Freiheit gesetat, dio ihrerseits 1,96 ceun Y $/ 2-N,-H C l$ binden. Durch Addition des gefundenen und des errochnetert Wertes ergibt sich dis zur Verseifung wirklich crforderliche Menge $1 / 2$ N.-Lösung $=3.91$ cern $1 / 2-\mathrm{N}$ - KOti. Berechnet fuir die Dibenzoylverbindung: $3.90 \mathrm{cem} 1 / 2-\mathrm{N}$.-KOH.

Aus dem Reaktionsgemisch ließ sich das Ausgangsmaterial wiedergewinnen, das unverändert den F. $76^{0}$ zeigte. Eine Unlagerung, analog der bei der Acetylierung beobachteten, war somit bei der Benzoylierung nicht erfolgt. Damit im Einklang steht die Beobachtung, daß der Schmelzpunkt der zum Vergleich aus der Base rom F. $113^{\circ}$ dargestellten Benzoylverbindung bei $118-119^{0}$ gefunden wurde.

Das isolierte Benzoylierungsprodukt der bei $76^{\circ}$ schmelzenden Base bestand somit aus Dibenzoylmethylamidoäthylphenylcarbinol. das auch bezüglich seines Schmelzpunktes den. Derivaten der natürlichen Basen nahesteht:

Nach den Angaben Mill e r s bildet die Dibenzoylverbindung des Ephedrins weiße, säulenförmjge, in Wasser unlösliche Krystalle vom F. 115/116 ${ }^{\circ}$. Das entsprechende Derivat des Pseudoephedrins schmilzt bei $119 / 120^{\circ}$ (L a d e n b u r g - O els ch läg el).

War so die Verbindung schon als das gesuchte Reaktionsprorlukt der Zusammensetzung $\mathrm{C}_{10} \mathrm{H}_{15} \mathrm{NO}$ charakterisiert, so blieb 
noch immer die verschiedene Löslichkeit des salzsauren Salzes dieser Darstellung und des 1914 beschriebenen Chlorids ungeklärt. Auch diese Unstimmigkeit sollte bald ihre befriedigende Erklärung finden (s. u.).

Zuvor mußte jedoch durch Darstellung und Charakterisierung der Platin- und Gold-Doppelsalze die Verbindung einmal weiter idestifiziert und zum anderen auf jhre Beziehungen zu den natürlichen Basen, denı Ephedrin und dem Pseudoephedrin, geprüft werrlen.

$$
\text { Platin-Doppels a I \%. }
$$

Die aus dem Hydrochlorid dargestellte Platinverbindung schied sich leicht aus mäßig konzentrierter Lösung in gelbroton Nadeldrusen aus, die bei $183^{\circ}$ unter Aufschäumen schinolzen. Auch diese Verbindung besaß den berechneten Platingehalt:

$1.1757 \mathrm{~g}$ Platin.

1. $0.6594 \mathrm{~g}$ des bei $100^{\circ}$ getrocknoten hiterials liejerten

E. $0.7584 \mathrm{~g}$ des bei $100^{\circ}$ gotrocknotan liaterialis listerten $0.1994 \mathrm{~g}$ Platin.

$$
\begin{aligned}
& \text { Berechnet fü } \\
& \left.\left(\mathrm{C}_{10} \mathrm{H}_{15} \mathrm{NO}\right)_{2} \mathrm{H}_{2} \mathrm{PtC}\right]_{6} \text { : } \\
& \text { (houmdert: } \\
& 26,64 \% \quad 26.29^{\circ}
\end{aligned}
$$

Das aus dem Filtrat dieser Bestimmung erhaliene salzsaure Salz bildete stark lichtbrechende, farblose rhombische Tafeln rom F. $187^{\circ}$.

Im Gegensatz hierzu stellte das früher (l. c.) gewonnene Platinsalz spitze rote Krystalle vom Schmelzpunkt 191 "S dar. Das PlatinDoppolsal\% des Ephedrins bildet blaßgelbe Nadeln vom l\%. 1860, lias des Psendo-Ephedrins scheidet sich ölig aus und wird durch Wasser zersetzo (li a d e n b u r g und O e l s a h läg e l).

$$
\text { Gold chlorid-To op pelsa } 1 z \text {. }
$$

Auch das Goldchlorid-Doppelsalz wich in seinem Verhalten von den früheren Beobachtungen ab. Die neue Verbindung lieferte runächst ebenfalls nur ölige Abscheidungen, diese konnten jedoch durch Lösen in Alkohol und Zugabe von Salzsäure unter der Einwirkung der Winterkälte in glänzende goldgelbe Nadeln vom $\mathbf{F}$. $107^{\circ}$ (1. Krystallisation) übergeführt werden. Ihr Goldgehalt lag etwas zu hoch:

$0.8950 \mathrm{~g}$ vorloren im Exsikkator $0,0115 \mathrm{~g}$ an Gewicht.

$0.8835 \mathrm{~g}$ der exsikkatortrockenen Verbindung lieferten $0.3493 \mathrm{gAu}$. Berechnet für $\left(\mathrm{C}_{10} \mathrm{H}_{15} \mathrm{NO}\right) \mathrm{HAuCl} \mathrm{H}_{4}$ : Gofund

$$
\text { Au } 39.11
$$

Als zweite Krystallisation resultierten säulenförmige durchsichtige Krystalle, die bei $113^{\circ}$ unter Gasentwicklung zu einer trüben Flüssigkeit schmolzen. Ihr Goldgehalt nähert sich dem berechneten Wert bis auf $0,23 \%$. Ein Gewichtsverlust im Exikkator konnte nicht beobachtet werden.

$0,3887 \mathrm{~g}$ lieforten $0.1529 \mathrm{~g}$ Gold.

$$
\begin{array}{cc}
\text { Berechnet: } & \text { Gefunden: } \\
\text { Au } 39,11 & 39,34^{\circ} \%
\end{array}
$$


Weitere Krystallabscheidungen schmolzen bei $115 / 118^{\circ}$. lieferten aber keine klare Schmelze. Von einer Analyse wurde daher Abstand genommen.

Im Gegensatz hierzu schmolz die 1914 dargestellte Verbindung, die sich ebenso zunächst ölig ausgeschieden hatte, bei $126^{\circ}$, nachdem bereits bei $120^{\circ}$ ein Erweichen eingetreten war. Da indessen das Aurat seinerzeit nicht rein erhalten worden war (der Goldgehalt wurde um $1,3 \%$ zu hoch gefunden), hat die Gegenüberstellung beider Verbindungen nur beschränkten Wert.

Das Golddoppelsalz des Pseudoephedrins bildet lange, verzweigte Nadeln vom F. $124^{\circ}$ (Ca ll i e $\beta$ ).

\section{Freies Methylamidoäthylphenylcarbinol.}

Waren bisher die Differenzen in dem Schmelzpunkt der verglichenen Verbindungen nicht nennenswert groß, so ergaben sich echt erhebliche Unterschiede bei dem Vergleich der freien Basen.

Das seinerzeit aus dem Platindoppelsalz isolierte Methylamidocarbinol bildete weiße verfilzte Nadeln, die bei $114-115^{\circ}$ schmolzen. In Uebereinstimmung damit wurde der Schmelzpunkt der aus der Acetylverbindung isolierten freien Base bei $113^{\circ}$ gefunden. Ebenso stimmte das Aeußere dieser Verbindung, die in weißen, seidenglänzenden Nadeln als Wandbelag resultierte, völlig mit obigem Material überein.

In Gegensatz hierzu resultierte aus der Behandlung des bei $184^{\circ}$ schmelzenden, gut krystallisierten salzsauren Salzes mit Soda und Aether eine zunächst ölige Masse, die beim Stehen krystallinisch wurde. Durch wiederholtes Umkrystallisieren aus Aether, dann aus Petroläther wurde die Verbindung in Gestalt filziger Nadelrosetten gewomen, die den bereits früher beobachteten aromatischen, nicht näher definierbaren Geruch in stärkerem Maß aufwiesen als die Base vom F. $113^{\circ}$. Auffallender weise schmolz die Verbindung aber schon bei $76^{\circ}$, selbst nach erneutem wiederholten Umkrystallisieren. Auch eine aus reinstem salzsauren Salz durch Natriumcarbonat und Aether isolierte Base zeigte trotz wiederholten Umkrystallisierens denselben Schmelzpunkt. Aeußerlich unterscheiden sich beide Verbindungen nur dadurch, daß die niedrigschmelzende Base etwas leichter löslich ist und feinere und stärker verästelte Nadeln bildet, die wenig schwächer glänzen als die Verbindung vom F. $113^{\circ}$.

Da reines Chlorid als Ausgangsmaterial gedient hatte, und auch durch wiederholtes Umkrystallisieren aus den vei'schiedensten Lösungsmitteln der Schmelzpunkt keine Veränderung erfuhr, war die Möglichkeit einer Verunr einigung der freien Base ausgeschlossen. Die Reinheit des Materials ergab sich weiter durch die Isolierung der ebenso schmelzenden Base aus dem Filtrat der analytisch charakterisierten 2. Pt-Doppelverbindung, und zwar zeigten sowohl die ersten als auch die weiteren Krystallisationen übereinstimmend den F. $76^{\circ}$. Ebenso ließ sich diese Base wieder in das bei $183^{\circ}$ schmelzende, aus rhombischen Tafeln bestehende Salz überführen.

Einen ähnlichen, noch niedrigeren $\mathrm{F}$. $\left(60^{\circ}\right)$ gibt $\mathrm{F}$ o u $\mathrm{r}$ n e a u ür das auf dem Weg über das Phenylpropylenjodhydrin dargestellte 
treie Methylamidoäthylphenylcarbinol. Indessen dürfte wohl kein reines Material vorgelegen haben, da F o u r n e a den Schmelzpunkt des salzsauren Salzes bei $178^{\circ}$ fand. Im Einklang damit wurde der Schmelzpunkt der aus den letzten Krystallisationen des Hydrochlorids isolierten freien Base vor dem Umkrystallisieren bei $64-65^{\circ}$ gefunden. Ein weiterer Hinweis auf dje Identität der verglichenen Verbindungen ist durch den gleichartigen Geruch der Basen gegeben, der auch von Fourneau beobachtet wurde.

Im direkten Gegensatz zu diesen Beobachtungen war andererseits der Schmelzpunkt der aus der Acetylverbindung (nach der Entfernung des Säurerestes) resultierenden freien Base bei $113^{0}$ gefunden worden. Auch lieferte dieses hochschmelzende Methylamiclocarbinol kein leicht krystallisierendes Hydrochlorid.

Dieser auffallende Unterschied in den Schmelzpunkten der beiden Basen (fast $40^{\circ}$ Differenz) läBt sich nur erklären durch die Annahme einer Umlagerungsfähigkeit, wie sie von $\mathrm{E}$. $\mathrm{S} \mathrm{ch} \mathrm{mid} \mathrm{t}$ für die natürlich vorkommenden Alkaloide (Ephedrin $\rightleftarrows$ Pseudoephedrin) experimentell festgestellt und wie sie von $\mathrm{C}$ a ll ie 3 für die Umwandlung Ephedrin $\rightarrow$ Pseudoephedrin speziell durch Acetylierung bewiesen worden ist. Wenn aber die Acetyliernng auch bei der synthetischen Verbindung in diesem Sinne gewirkt hatte, so war auch anzunehmon, daß diese sich gegen andere Agentien, speziell gegen Salzsäure, ebenso verhalten mußte wie die natürlich vorkommenden Basen.

\section{Umlagerungs sversuche.}

\section{Verhalten des synthetischen IIydrochlorids gegen konzentrierte Salzsäure.}

Analog einem von E. S $\mathrm{eh} \mathrm{midt}^{1}$ ) bei der Umwandlung des Ephedrins in das Pseudoephedrin angewandten Verfahren wurde $1 \mathrm{~g}$ des gut krystallisierten salzsauren Methylamidoäthylphenylcarbinols mit der zehnfachen Menge 25\%iger Salzsäure im Pohr eingeschlossen und 12 Siunden lang im Wasserbad erhitzt.

Beim Verdunsten der schwach gelblich gefärbten Lösung resultierte zunächst unverändertes Chlorid, gekennzeichnet durch die gute Krystallisierbarkeit, die rhombische Form und den F. $183^{\circ}$ sowie durch Ueberführung in die freie Base, die, wie das Ausgangsmaterial, bei $76^{0}$ schmolz.

In Anbetracht der leichten Löslichkeit des früher aus der hochschmelzenden freien Verbindung hergestellten salzsauren Salzes mußte sich - falls überhaupt eine Ümlagerung stattgefunden hatte - das Hydrochlorirl der umgelagerten Verbindung in den Mutterlaugen befinden. Diese wurden daher in die freie Base übergeführt.

Bei dem Zusatz von Sodalösung blieb die Flüssigkeit klar. Aether entzog ihr noch beträchtliche Mengen einer freien Base, die sich beim Verdunsten des Lösungsmittels als ein aus dünnen, verfilzten Nadeln bestehender Wandbelag, der durch ölige Beimengungen

1) Arch, d. iharm, 244 (1906), 239. 
verumreinigt war, abschied. Nach dem Abpressen und Unkrystallisieren zejgte djese Verbindung den. Schmelzpunkt $76^{\circ}$ und er wies. sich somit als unverändertc 8 Mater ial.

Auffallenderweise erfuhr die noch siark alkalische Lïsing, die ań Aether nach viermaligem Ausschücteln nichts mehr abgab, durch Zusatz von konzentrierter Natronlauge eine starke, milchige Trübung, die beim Durchschïiteln mit Aether von diesem aufgenonmen wurcle. Bei dem Veadunsien der Aetherlösung resultierten sofort leicht krvstallisie ende seidenglänzende Nadeln in Gestalt eines dichten zusammenhängenden Wandbelags, die bei 112 - $115^{\circ}$ schmolzen und sich bei dem Umkrystallisieren aus Aether als schwerer löslich erwiesen als die Verbindung vom $F .76^{\circ}$.

Der Versuch hatte also bewiesen, daß in der Tat das svnthetische Produkt auch dureh Behandeln mit Salzsäure eine U'mwandlung erfährt im Sinne der Umlagerung des Ephedrins in das Pseudoephedrin, und zwar hatte sich auch hier die Reaktion nur bis zu einem gewissen Grade vollzogen, ähnlich wie sich auch Ephedrin und Pseudoephedrin nur bis zur Erreichung eines Gleichgewichtszustandes umlagern lassen.

Andererseis hatte der Versuch im Zusammenhang mit den früheren Analysen weiter aber auch bewiesen, daß sowohl der Base vom $F$. $76^{\circ}$ als auch der bei $113^{\circ}$ schmelzenden Verbindung die Formel $\mathrm{C}_{10} \mathrm{H}_{1:}$ NO zukommt. Der Kürze halber soll im folgenden das niedrig schmelzende Methylamidoäthylphenylkarbinol als Base A, das ho ch schmelzende Produkt als Base B bezeichnet werden.

Wenn nun unter der Finwirkung der Salzsïure die Umlagerung der Base $A$ in die Base B stattgefunden hat te, so war es auch denkbar, daß dieselbe Reaktion bei dem Eindampfen der dur.h katalytische Hydrierung erhaltenen wässerigen Iösung eingetreten sein konnte. Auch hier war ja ein Zusat $z$ von verdünnter Salzsäure gemacht worden, die bei dem Einengen der Tüsung natürlich konzentrierier werden mußte. Vorausgesetzt, daß die Umlagerung überhaupt bei gewöhnlichem Druck stat fand, mußten anch die hierbei resultierenden Mutcerlaugen die hochschmelzende Base enthalten.

Auch aus diesen Mutterlaugen wurde zunächst durch Ausschütteln mit Soda und Aether eine Base von Trpus A neben öligs bleibenden Anceilen isoliert. Bei dem nach imehrfachem Ausschüt:eln mit Aether erfolgenden Zusatz konzentrierter Natronlauge zeigte sich dieselbe Firscheinung wie bei obigem Versuch: auch hier trat eine milchige Trübung auf. die ron Aether aufgenommen wurde. Aus dieser Lösung krystallisierte dann in der Tat die Base $B i_{u}$ typischen sejdenglänzenden zusammenhängenden Nadeln als Wandbelag.

Neben diesen beiden Formen resultierte schließlich bei dem Umkrysiallisieren der weniger gut. ausgebildeten und der ölig gebliebenen Anteile aus Chloroform ein weißes Pulver, das bei den Erwärmen der chloroformhaltigen ätherischen Lösung an Menge zunahm, so daß es den Anschein hatte, als ob noch eine dritte isomere Base vorläge. Diese, selbst in Aether unlösliche Verbindung zeigte den abnorn hohen F. 1840. Dieselbe erwies sich inclessen 
bei weiterer Prüfung als Hydrochlorid, entstanden durch Chlorabspaltung aus dem (hioroform.

Nun wäre es immerhin denkbar gewesen, clab auch das gut krystallisierende Hydrorhlorid der Base A beteits beide Formen enthielte, da die Base $B$ ja nur clureh Alkalisieren der vorher mil Soda und Aether ausgeschüttelten Isösung mit Natronlange hat te isoliert werden können. Tm auch hierüber Klarheit zu schaffen, wurde das Hydrochlorid rom F. $184^{\circ}$ ebenfalls zunächst nit Sodalisung und Äether behandelt und dann mit Narronlauge versetzt. In. diesem Fall trat jedoch keine milchige Trübung der alkalischen Flüssigkeit ein, und ebensowenig vermochte Aether dem Reaktionsgemisch weitere Mengen einer Base zu entziehen. Die mit Soda ind Aether in Freiheit gesetzte Base schmoli, wie erwartet, bei $76^{\circ}$.

Um schließlich zu beweisen, daß das Alkalisierungsmittel whe Einfluß auf den Schmelzpunkt der Base war, wurde das salzsaure Salz vom (F. 1840) direkt mit Natronlauge and Aether ansreschütelt. Indessen resultierte arch jn diesem Fall nur Base A. rom F. $76^{\circ}$.

Auch dieser Versuch hatte somit gezeigt, daß eine Umlagerumg vormutlich von Base $A$ in Base $B$ auch unier gewöhnlichem Atmosphärendruck stattgefunden haben muB.

Andererseits konnte festgestellt werden, daB die Umlagerung Ler Base A in Base B durchaus nicht leicht erfolgt. Weder' durch langdauerndes Erhitzen and wiederholtes Findampfen der Base A in it Aether, Aceton, Petroläther, Ligroin oder Alkohol verschiedener. Innzentrationen, noch auch mit Gomischen dieser Lösungsmittel komnte Base B gewonnen werden. Ebensowenig Erfolg brachte lingeres Erhitzen mit (hloroform, das (s. o.) zur Bilduns des salzsaruen Salzes der Base A führte.

Bei dem Versuch, die Base A direlt im Wasserbad durch Wrhitzen umzulagern, verflüchtigte sie sich völlig und zwar unzersetzt. Durch Bedecken der Gefäße konnte die Base unverändert als strahlig-krystallinisches Sublimat vom $\mathrm{F}$. $76^{0}$ wieder gewonnen werden.

Ebensowenig lies sich eine Umlagerung, durch Behandeln mit. Natronlauge erzielen. Sowohl nach dreitägigem Stehen bei Zimmerremperatur mit 10-und 30\% iger Lauge als auch nach zweistündigem Erhitzen im Wasserbad blieb die Base völlig unverändert.

Nach der so festgestellten Umlagerunosfähigkeit der Base A in Base B, die sich mit dem Verhalten des Ephedrins völlig deckt (auch dieses ließ sich durch Alkalien and Eihitzen mit Wasser und inderen Lösumssmitteln nicht umlagern), erschien es angebracht, lie beiden synthetischen Verbindungreformen der Formel $\mathrm{C}_{10} \mathrm{H}_{1:} \mathrm{NO}$ in (Aestalt ihrer Chloride bzw. Doppelsa!ze einander gegenüberzustellen, zumal jetzt gut gekennzeichnetes reines Material zur Ver fügung stand.

Unter Finhaltung derselben Versichsbedingungen wurden ranächst

\section{die salzsauren Salze der isomeren Basen}

largestellt. Während bei den freien Verbindungen (s. o.) der Sthmelzpunkt der Base A niedriger lag als derjenige der Base $B$. liogen die Verhälinisse bei den salzsauren Salren gerarte ungekelut. 


\section{A. Eberhard: Inaktives Ephedrin bez. Pseudoephedrin.}

Das salzsaure Salz der Base A ist bereits oben beschrieben. Es lrrystallisier:- lejcht aus alkoholischer oder salysaurer Iösung in Gestalt farbloser rhombischer Tafeln vom F. 184. Bei dem über das Platinsalz gereinigten Präparat wurde der Schmelzpunkt bei $187^{\circ}$ gefunden. Einen thnlichen Wert (190\%) fand $B$ ü $m$ in i $g^{1}$ ) für das aus Phenylpropylenoxyd dargestellte salzsaure Methylamidoäthylphenylkarbinol, das ebenfalls in Gestalt rhombischer Tafeln resultierte. Four nea $u^{2}$ ) gibt ils Schmelzpankt der ähnlich dargestellten Verbindung $178^{\circ}$ an. Da die von ihm hieraus darestelte freie Base nu den Schmelzpunkt $60^{\circ}$ zeigte, dürfte seinen Beobachtungen wohl ein von. Veranreinigungen nicht ganz freies Präparat z.ıg' ande gelegen haben.

Das bei meinen früheren Versuchen nur in selır kleiner Menge $(0,03 \mathrm{~g})$ isolierte salzsaure Salz der Base B bestand aus weiBen Warzen und ebensolehen Flocken, die beide bei $180-181^{\circ}$ suhmolzen. Duich seine Jeichte Iöslichkeit unterscheidet es sich erheblich von dem obigen Salz der Base A. Auch die erneute Darstellung dieser Verbindung aus der Base B ergab ein sehr leicht lösliches Produkt, das erst aus den letzten sirupartigen Tropfen der Lösang zu Nadolrosetten erstarte und mit Hilfe von Aceion von anhaftenden fremden Bestandteilen befreit wurde. Trotz dieser äußeren Uebereinstimmungen lag der Sohmelzpunkt bei $153-154^{\circ}$. Da nu! eine kleine Menge der Base B zur Verfügung stand, wurde von einer weitcren Reinjoung zunächst Abstand genommen. Erst spätro gelang es, diese Verbindung über das Platin- und Goldsalz weiber zu reinigen (s. u.). Aber auch so stieg der Schmelzpunkt der in seidenglinzenden, aus haarfeinen Nädclchen bestehenden Büschel nicht über $158-161^{\circ}$. Der Widerstreit mit den fü̈heren Augaben ist nur so zu erklären. daß aach damals beide Formen vorgelegen haben, dnß aber rlie minimale Menge des Materials das Vorhandensein zweier verschiednner Salze nicht zu erkennen gestatete. Da jedesmal nur dic erste Krystallisation bzw. Fallung zur Bestinmung des Schmelzprnktes benutz wurde, ist nur das hochsehmelzencle Hrdrochlorid der Base A bei den Angaben berücksichtigt wonden. Dä sich die freien Basen beziglich ihrer Löslichkeit umgekehyt verhalten, so ist dementsprechend die schwerlösliche Base B ruerst allskrystallisiert und somjit die erste Krystallisation vom $\mathbf{F}$. $114^{\circ}$ bis $115^{\circ}$ als Base des bej $191^{\circ}$ schmelzenden salzsauren Salzes angesprochen worden.

\section{Platindoppelsa $1 \%$ e.}

Zur weiteren Charakterisientung wurken die salzsauren Salze der beiden Basen in. ihre Plainsalze übergeführt.

Base A lieferte gelbrote Roscten, ans durchsiehtigen flachen Nadeln und Blattchen bestehond, dje bei $183^{\circ}$ mncer Aufsehäumen schmolzen. Ihre Identiat mit dom gesnchten Reaktionsprodukt ist bereits oben dureh Bestimmumo les Platingehaltes bewiesen worden. Line erneute Bestimmany bostatigue dieses Resultat.

1) Inaug.-Dissert. Marburg 1909.

s) Journ. d. Pharm. et de Chim. XX. (1904), $481 \mathrm{ff}$. 
A. Eberhard: Inaktives Ephedrin bez. Pseudoophedrin. 125

Im Gegensatz zu dem Platinsalz der Base B enthält dieses Doppelsalz kein Krystallwasser.

$0.1196 \mathrm{~g}$ (bei $100^{\circ}$ getrocknet) ergaben $0.0318 \mathrm{~g}$ Platin.

Berechnet fiir $\left(\mathrm{C}_{10} \mathrm{H}_{15} \mathrm{NO}\right)_{2} \mathrm{H}_{2} \mathrm{P}^{2} \mathrm{Cl}_{6}$ : Gefunden :

$$
\text { Pt } 26,37
$$

Das der Base B zugehörige Platinsalz schied sich zunächst ölig aus, ging aber bei längerem Stehen allmählich in den krystallisierten Zustand über. Es bildete rotgelbe, undurchsichtige, derbe Warzen, die bei $177^{\circ}$ unter Aufschäumen schnolzen.

$0,2759 \mathrm{~g}$ verloren im Wassurtrockenschrank $0.0078 \mathrm{~g}$ an Gewicht. Berechuet fiur $\left[\left(\mathrm{C}_{10} \mathrm{H}_{15} \mathrm{NOO}\right)_{2} \mathrm{Hi}_{2} \mathrm{PtCl}_{6}+\mathrm{H}_{2} \mathrm{O}\right]$ : Gefunden:

$$
\mathrm{H}_{2} \mathrm{O}=3,38 \quad 2,83 \%
$$

Platin.

$0.2481 \mathrm{~g}$ dos bei $100^{\circ}$ getrockneten Platinsalzes orgaben $0,0656 \mathrm{~g}$

$$
\begin{aligned}
& \text { Bereclinet für }\left(\mathrm{C}_{10} \mathrm{H}_{15} \mathrm{NO}\right)_{2} \mathrm{H}_{2} \mathrm{PtCl}_{6} \text { : Gefunden: } \\
& \text { Pt } 26,37 \quad 26,44 \%
\end{aligned}
$$

Auch der Schmelzpunkt dieser Verbindung liegt wesentlich niedriger als der 1914 für diese Verbindung gefundene. Damals resaltierten spitze rote Krystalle vom F. 1910, allerdings neben gelben Nadeln vom F. 146 ${ }^{\circ}$. Ob der Unterschied durch den Krystallwassergehalt der neu isolierten Verbindung bedingt ist, mag dahingestellt bleiben. Tndessen kann aus den oben angegebenen Gründen damals ebensogut ein Doppelsalz der Base A vorgelegen haben. Fourneau (1. c.) kennzeichnet seine Platinverbindung als sehr lei ht löslich in Wasser. - Das Platinsalz des Ephedrins billet blaßgelbe Nadeh vom F. 186 , kommt also hier zum Vergleich nicht in Frage. Dagegen zojgc dieses synthetjsche Platindoppelsalz ebenso wie das Hydrochlorid sehr nahe Bezichingen an den entsprechenden Verbindungen des Pseudoephedrins, dessem Platin-

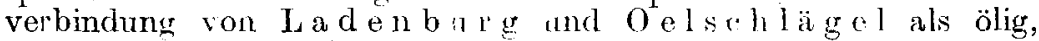
durch Wasser zersetzbar, bezeichnet wird.

Beide Doppelverbindungen entspraches somit der Formel $\left(\mathrm{C}_{10 \mathrm{H}}{ }_{15} \mathrm{NO}\right)_{2} \mathrm{H}_{2} \mathrm{PtCl} \mathrm{C}_{6}$. Auch hier hebt sich clas Platinsalz der Base $\mathrm{B}$ von dem der Base $A$ erheblich ab.

$$
\text { Gold op pelsalze. }
$$

Aue:. die Golddoppelverbindungen der Basen wurden zur weiteren Idencifizierung herangezogen. Um das Verhalten dieser Aurate nebeneinander genaa boobachten zu können, wurden das aus einer früheren Platinbestimmung (s. o.) gewonnene, bej $187^{\circ}$ schmelzende Hydroshlorid der Base A sowio die Filtrate obiger Platinbestimmungen als Ausfangsmaterial benutzt.

Beide Goldsalze schieden sich zunächst ölig ans, konnten aber durch Wiederaufnehmen mit eben ausreichenden Mengen Wassers und langsames Verdunsten der Lösungen im Exsikkator zur Krystallisation gebracht werden. War diese cinmal angeregt, was leicht durch Impfen zu erreichen war, so kounte durch Einstellen in den Vaknumexsikkator die Krystallisation beschleunigt werden, ohne daß sich ölige Produkte bildeten.

Das der Base A zugehörige Goldsalz resultierte zunähhst in Gestalt rechteckiger dünner Tafeln von gelber Farbe, dann im 
Vakuumexsikkator in Form goldglänzender Lamellen. Beide Formen schmolzen übereinstimmend bei $115^{\circ}$, eine Mischung beider zeigte keine Depression.

$0,3373 \mathrm{~g}$ exsikkatortrockenen Goldsalzes lieferten $0.1332 \mathrm{~g}$ (iold.

Berechnet für $\mathrm{C}_{10} \mathrm{H}_{15} \mathrm{NO} . \mathrm{HAuCl}_{4}$ : Gefunden: $\mathrm{Au} \mathrm{39, \textrm {ll }}$ $39,19 \%$

Weiter erhaltene zitronengelbe flache Nadeln von starkem Glan\% zeigten einen sehr naheliegenden Schmelzjunkt (115--1160). Gold.

$0,1086 \mathrm{~g}$ des exsikkatortrockenen Goldsalzes lieferten $0.0428 \mathrm{~g}$

$$
\begin{array}{cc}
\text { Berechnet : } & \text { Gefunden : } \\
\text { Au 39.11 } & 39.41 \%
\end{array}
$$

Das Filtrat der Platinbestimmung der Base B lieferte das am stärksten gefärbte Goldsalz in Gestalt rötlichgelber flacher glänzender Nadeln vom F. 115-116 ${ }^{\circ}$.

$0,2414 \mathrm{~g}$ des exsikkatortrockenen Doppelsalzes ergaben $0,0947 \mathrm{~g} \mathrm{Au}$.

$$
\begin{array}{cc}
\text { Berechnet: } & \text { Geînden : } \\
\text { Au 39.11 } & 39.23^{\circ} ;
\end{array}
$$

Die Goldsalze waren also weder in ihrer Form, noch in ihrem Goldgehalt wesentlich von einander verschieden. Der einzige Unterschied bestand in der dunkleren Färbung des Aurates der Base B.

Das früher (1914) gewonnene Doppelsalz hatte zwar höher. geschmolzen (F. 126" nach vorhergehendem Erweichen bei 120\%), indessen war das Aurat unrein, wie aus seinem um 1,3\% zu hohen Goldgehalt hervorging. Ein Vergleich mit diesem Produkt ist also ohne Bedeutung. Aber auch den Auraten der natürlichen Basen ähnelt keines der Doppelsalze, selbst nicht das, der Base B. Pseudoephedringoldchlorid bildet lange, verzweigte, bei $125-126^{\circ}$ schmelzende Nadeln.

Ein Vergleich mit dem ron $\mathrm{F}$ o u $\mathrm{r}$ n e a $u$ hergestellten Goldsalz derselben Zusammenseizung ist gänzlich unmöglich, da dieser das Aurat nur in öliger Form erhalten hat.

Um schließlich zu ergründen, ob nicht bei der Ueberführung der Basen in ihre Boppelsalze eine ernetie Unlagerung eingetreten war - diese Möglichkeit war hei der fast völligen Uebereinstimmung der Goldsalze nicht von der Hand zu weisen - wurden die Filtrate obiger Goldbestimmungen zur Zurückgewinnung der salzsauren Salze getrennt eingeengi und schließlich aus diesen die freien Basen isoliert.

Das Filtrat des Godlsalzes A krystallisierte unverändert leicht in farblosen, lichtbrechenden. vielseitigen Tafeln vom F. $187^{\circ}$ wie das Ausgangsmaterial. Ehenso schmolz die hieraus isolierte freie Base wieder bei $76^{\circ}$.

Aus dem Filtrat des Aurates B konnten dagegen direkt keine Krystallisationen erzielt werden. Ers der ölige Verdunstungsrückstand erstarte allmählich $\%$ einer strahlig krystallinischen Masse, die sich in siedenden Aceton nur schwer löste. Bei dem Erkalten schied sich ein Wandbelag von haarfeinen, seidenglänzenden Nadeln ab, die bei 158-1610 schmolzen. Ta die Accetonlösung beim Siehen an der Iuft Feurhígkeit aufnahm, erstarte die 
Hanptmenge wieder nur alıs den letzten höshsthonzentrierten Isaugen. Erst als der ernellt mit heißem Aceton aufgonommene Rückstani im Fxsikkator zur Krystallisation gebracht uurde, resultien ie die gesamte. Menge in Form seidenglänzender, verfilzter Nadelbüschel desselben Schmelzpunktes. Da die hieraus isolierte freic Basc den $\mathbf{F}$. $114^{0}$ zeigte, sn war damit erwiesen, daß anch hicr keine Rückverwandhung in Base A cingetreten war.

Bei dem Jurchschütteln mi: Soda und Lether schied sich die freie Base A ölig aus. In der Anmahme, daß vielleicht das ïlige Produkt durch Impfrn in jede der gewünschten Formen übergehe'l kïnne, wurde eim. Sipur der bei $114^{\circ}$ schmelzenden Base zugefïgt. Der Amualime zuwider erstarte jedoch der ölige Verdunstungsrückstand zu eimer harten weißen Mnsse, die vor und nach dren Emkrvstallisjeren den F, 76" zeigte. inaktiv:

il ie voraurousehen war, erwiesen sich beids Basen als op.isch

Theoretisth fehnt sich dit: Denlung der Basen A und b alle an dic der natürlichen Basen an. Aluch hier muB angenonnonen werden, dals ein Fill geometrischer fsomerie vorliegt, bedingi dureh das labile Verhalten der Hydroxylgruppe.

(Allerdings bleibt dor umgekehris Verlauf (Base $B \rightarrow$ Bass $A$ ) noch experimentell zu beweisen.) Nur wnter rlicoer Annahne liibt sich eino Wrklïrung dafür finden, daß3 durch Acetylierung rine vollstiondige Cmwandlung von $1 B_{\mathrm{is}} \mathrm{A}$ in Base 13 stattfindrel, wälrend bei cler Brnzovlierung die Base A unveräuder: blojbt.

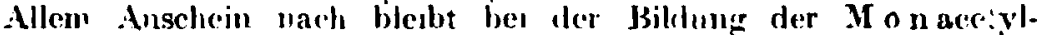
verhindung dir OH-(iruppe intakt und damit reaktionsfähig. währenil durch linwirkung ron Benzoylehlorid ein D j benzoyikarhinol resultiert, dessen OH-(iruppe dureh einen Benzovelrest verchlosisen ist, so. daß cine Limlagerung unterbleibt. (Einen analogen Fall aus cler Jiteratur vermag ich allerdings nicht anzı. geben.) I m linklang mit dieser Annahme oler Bestäudigkeit beider Basen yegen BenzoyldhJorid zeigt die entsprechende Jenzoyl. verbindung der Base 13 einen un jo höheren Schmelzpunkt als die benzoylierte Base A. Dieselbe Tempermurdifferenz zeigen die Dibenzoyfderivato des Fphedrins bzw. Psendoephedrins.

Im vorsichenden zweiten Tol der Abhandlung ist somit gezeigt worden, daß neben der früher dargestellten Base vom F. $14^{\circ}$ (Base B) eine zueite stabile Form des Methylamidoäthylphenylkarbinolsi ion $F$. $76^{\circ}$ (Base $A$ ) existiert, die sich auch in ihren Salzen von der früher beschriehenen Verbindung der $/ 1$. sammensetzung $\mathrm{C}_{10} \mathrm{H}_{1 \mathrm{NO}} \mathrm{NO}$ unterscheiclet. Weiter haben die Ver. suche gelehrt, daß die synthetischen Verbindungen bzw, deren Salye und Doppelverbindungen in ihrem Aeußeren und jhrem Schmelzpunkt gewisse Achnlichkeiten mit den naturcllen Basen anfweisen; besonders tritt dies berüglich der Base B und des Pscudoephedrins zutage. Bezüglich der Cimwandelbarkejt der Base A in Base 13 crgab sich sogar völlige T'cbercinstimmung mit Fydıedrin bzw. Pseudoephedrin: und zwar vermay genau wie bei dem Ephedrin auch bei cliesen Verbindungen Salzwïure schon unter gewöhnlichen 1) ruck. be'seer im Einsithmelzrohr bei hoher Temperatur, die Um. 
128 A. Eberhard: Inaktives Ephedrin bez. Pseudoephedrin.

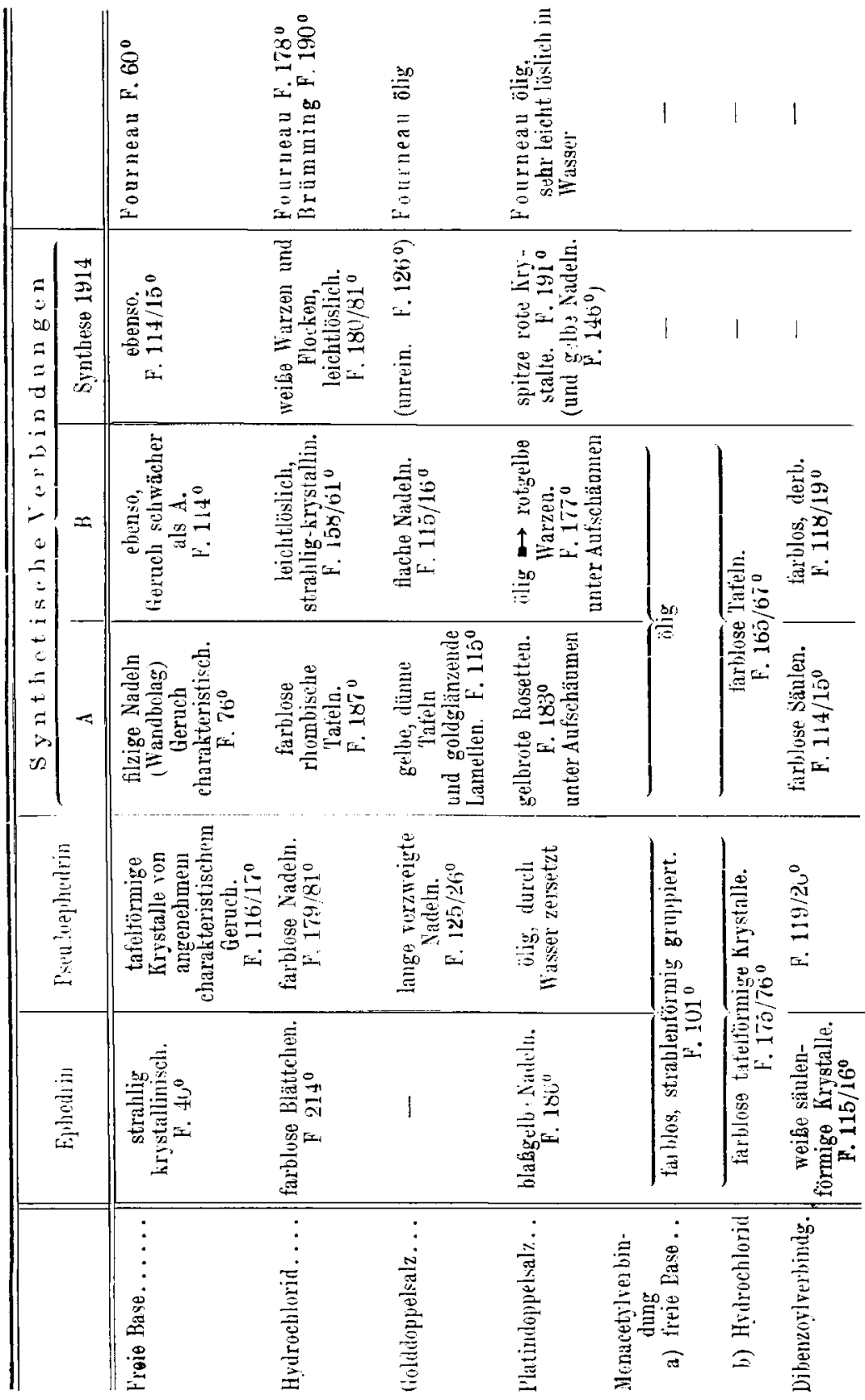


A. Eberhard: Inaktives Ephedrin bez. Pseudoephedrin. 129

lagerung partiell hervorzurufen, während durch Acetylierung Base A vollständig in Base B übergeht. Diese Einwirkung wird dagegen durch Benzoylierung nicht erzielt (s. o.). Ebensowenig erfolgt durch trockenes Erhitzen der Base A oder durch Frwärmen mit Alkalien oder mit einer Anzahl von Lösungsmitteln eine Umlagerung. Bei dem Erhitzen mit Chloroform resultiert infolge einer Zersetzung des Lösungsmittels das salzsaure Salz.

Da die Verbindungen demnach bezüghch ihrer Umlagerungsfähıgkeî̀ mit den natürlichen Basen völlige Uebereinstimmung zeigen, so ist durch die vorliegenden Versuche die Synthese des. inaktiven Ephedrins bzw. Pseudoephedrins, die sich bisher aus den naturellen Basen nicht darstellen ließen, in befriedigender Weise realisiert, so daß jetzt auch Versuche zu deren Spaltung ermöglicht sind.

Eine Uebersicht über die Beziehungen zwischen Ephedrin, Pseudoephedrin und den synthetischen Verbindungen gibt vorstehende Tabelle.

Zum Schluß sei es mir gestattet, meinem hochverehrten Marburger Lehrer, Herrn Geheimen Regierungsrat Professor Dr. E. S c hm i.d t, für die Ueberlassung des Gebietes sowie für seme mir stets in reichstem Maße erwiesene Anregung und Förderung bei der Durchführung der vorliegenden Arbeit meinen verbindlichsten Dank auszusprechen. Ebenso bin ich clen Herren Professor Dr. L. W öhler, Geheimen Obermedizinalrat Protessor Jr. G. H e y 1 und Professor Dr. F in g e r an der Technischen Hochschule zu Darmstadt für die stets wohlwollende Unterstützung meiner Arbeiten nach meiner Uebersiedelung nach Darmstadt zuk sufrichtigem Dank verpflichtet. 